\begin{tabular}{|c|l|}
\hline Title & Wrapped brane gas as a candidate for dark matter \\
\hline Author(s) & Sano, Masakazu; Suzuki, Hisao \\
\hline Citation & $\begin{array}{l}\text { Physical Review D, 81/2), 024042 } \\
\text { https://doi.org/L0.1103/PhysRevD.81.024042 }\end{array}$ \\
\hline Issue Date & 2010-01-15 \\
\hline Doc URL & http://hdl.handle.net/2115/42807 \\
\hline Rights & ○2010 The A merican Physical Society \\
\hline Type & article \\
\hline File Information & PRD81-2_024042.pdf \\
\hline
\end{tabular}

Instructions for use 


\title{
Wrapped brane gas as a candidate for dark matter
}

\author{
Masakazu Sano and Hisao Suzuki \\ Department of Physics, Hokkaido University, Sapporo, Hokkaido 060-0810 Japan
}

(Received 26 July 2009; published 28 January 2010)

\begin{abstract}
We consider brane gas models based on type II string theories and analyze the mass, the RamondRamond charge, and the charge on moduli fluctuations of branes wrapping over cycles of a compactified space in the four-dimensional Einstein frame. A six-dimensional torus and Calabi-Yau threefolds are considered for the Kaluza-Klein reduction. A large volume of the compactified space and a weak string coupling gives rise to point particles of the wrapped branes which have a light mass and a small charge of the Ramond-Ramond flux and of the moduli fluctuations, while the particles become very heavy in the string frame. We find that the masses and the charges satisfy the seesaw like dual relations which become time independent in the four-dimensional Einstein frame.
\end{abstract}

DOI: 10.1103/PhysRevD.81.024042

PACS numbers: 04.50.-h, 11.25.- w, 11.25.Mj

\section{INTRODUCTION}

The string gas model [1] is one of the interesting scenarios in string cosmologies (see [2-6] for recent reviews). The simplest of such models treats the space as $T^{9}$. If many strings wrap over cycles of a nine-dimensional torus, $T^{9}$ at a very early universe, the cosmological singularity can be resolved by $T$ duality [7]. The string gas models also provide an intriguing idea on a realization of the large three-dimensional space by annihilation of the strings, while the scale of the six-dimensional torus is stabilized by the tension of remaining strings. The brane gas models [8] are an extension of the idea and have been considered a cosmological role of windings of D-branes.

Although these gas models are very interesting, one of the serious problems is the moduli stability. This problem is a serious obstacle to build string/brane gas models consistent with a realistic phenomenology and cosmology. This is resulting from massless scalar fields that appear on the three dimensions without the moduli stabilization. There are many interesting works on the moduli stabilization [9-24]. Among the stability of many moduli fields, the stabilization of the dilaton field has been difficult in brane gas models. Recently, we have shown that electric fields on D-brane windings of NS5-brane and Kaluza-Klein monopole (KK5-monopole) can stabilize radial moduli fields of a six-dimensional torus $T^{6}$ and the dilaton field simultaneously in brane gas models based on type II string theories [25].

According to the result of [25], gases of branes wrapping over only cycles of $T^{6}$ make the energy density of a pressureless matter, namely, the energy density is inversely proportional to the third power of the scale factor. This implies that we observe those wrapped branes as point particles on the three-dimensional space. This fact leads to the appearance of the dark matter of the wrapped branes, because wrapped branes are not ordinary matters. The possibility of the dark matter of the wrapped branes has been investigated in various models. The appearance of light particles of the wrapped branes in the string frame, if the string scale, $\sqrt{\alpha^{\prime}}$ is larger than the Planck length in Ref. [26]. The large scale structure and the accelerating phase were discussed in [12,27-30] without an influence of the Ramond-Ramond (RR) flux, although D-branes have the RR charges. However, the mass and the charge of RR flux are not estimated simultaneously in the fourdimensional Einstein frame which is required for obtaining realistic models.

We take the four-dimensional Einstein frame, when we consider the time-independent four-dimensional gravitational constant after the Kaluza-Klein reduction. In this frame, the realization of light wrapped branes is not trivial, since it is known that a large volume of the six-dimensional torus and a weak string coupling give rise to heavy wrapped branes in the string frame for a small string length [31].

The purpose of this paper is to study wrapped branes in the brane gas cosmology based on type II string theories as dark matter, using the effective field theory of brane gas models. We estimate the mass and the charge on the RR flux and on moduli fluctuations. These fluctuations also provide forces between the branes. We find some models where branes can be realized as dust particles whose masses can be around $10 \mathrm{TeV}$ scales. We take the string scale as $1 / \sqrt{\alpha^{\prime}} \sim m_{\text {Planck }}$ and consider a compactification with $T^{6}$ and the Calabi-Yau threefold $\left(\mathrm{CY}_{3}\right)$, using the four-dimensional Einstein frame which is the key idea to obtain a light mass and a weak interaction.

The description of the effective field theory requires a large volume of a compactified space and a weak string coupling, because quantum corrections should be suppressed and the perturbative expansions should be valid. In the string frame, those conditions lead to the appearance of heavy wrapped branes, as the world volume action of the brane is proportional to its volume and the inverse of the string coupling. It seems apparent that the dark matter candidate of the wrapped branes may be difficult in this frame. However, in the four-dimensional Einstein frame, 
we will show that there are cases where the wrapped branes obtain the light mass and the small charge on the RR flux and on the moduli fluctuations under the large volume and the weak string coupling. We will show that the effective masses and charges satisfy the seesaw like dual relations which are time independent, while each quantity depends on the time variable through moduli fields. The dual relations are one of reasons for the existence of the various light wrapped branes with the small charges.

From the phenomenological point of view, the estimation of the mass and charge is mainly done by controlling scales of the compactified space by hand. However, in this paper, the string scale is of the order of the Planck scale and then the existence of light particles of wrapped branes is quite nontrivial under the large volume. In one model, we find that the mass of the D0-brane is of the order of $\mathcal{O}(10) \mathrm{TeV}$, if we take the six-dimensional compactified volume as $\left(\mathcal{O}\left(10^{5}\right) \times 2 \pi \sqrt{\alpha^{\prime}}\right)^{6}$ where $\sqrt{\alpha^{\prime}}$ is the string length and holds $m_{\text {Planck }} \sim 1 / \sqrt{\alpha^{\prime}}$ in this paper. The square of the effective charges of the RR flux and of the moduli fluctuations is of order $\mathcal{O}\left(10^{-30}\right)$ by the same scale of the compactification. Then, the D0-branes are a possible candidate for the dark matter at a late time.

We also consider the masses of wrapped branes by a D0KK5 brane gas system [25], because the system can be stabilized by the scale of the $T^{6}$ and the dilaton field. It is not trivial that both the weak string coupling and the large scale of the compactification are realized simultaneously in the specific model after the moduli stabilization. We find such realization of those conditions exists if the number density of D1 and KK5 satisfy a specific condition which is given later. In the case of the Calabi-Yau compactification, we cannot prove the moduli stabilization in this model, however the dual relation is also satisfied and then we can show that light particles are generated for the large volume of the $\mathrm{CY}_{3}$.

This paper is organized as follows. In Sec. II, we will give the definition of the four-dimensional Einstein frame. In Sec. III, we will consider the effective world volume action of wrapped branes in the four-dimensional Einstein frame. In Sec. IV, we will show the electric-magnetic dual relation between masses of the wrapped branes. The dual relation explains the existence of the light wrapped branes. In Sec. V, we will see a case in which the electric-magnetic dual relation of wrapped branes is satisfied in the $\mathrm{CY}_{3}$ compactification. In Sec. VI, we will investigate the effective RR charge in the four-dimensional Einstein frame. The effective coupling of the $\mathrm{D} p$ - and $\mathrm{D}(6-p)$-branes also satisfies the electric-magnetic dual relation. In Sec. VII, we will comment on the charge of fluctuations of the moduli fields, because those fluctuations also give interactions between various branes. In Sec. VIII, we would like to analyze an explicit model constructed by a D1-KK5 brane gas system. Section IX will be devoted to the summary and some discussions.

\section{FOUR-DIMENSIONAL EINSTEIN FRAME}

In this section, we shall define the four-dimensional Einstein frame which gives the time-independent fourdimensional gravitational constant after a compactification. In general, the Kaluza-Klein reduction gives rise to a coupling between the four-dimensional Einstein-Hilbert term and various moduli fields. If those moduli fields are functions of coordinates of the compactified space, we can integral out moduli fields and renormalize the factor into the higher dimensional gravitational constant. However, in cosmologies, moduli fields depend on the time and, therefore, we cannot integral out the moduli fields completely. The time-independent Newton constant can be easily realized in the four-dimensional Einstein frame which is useful to analyze a behavior of scale factors and various fields.

We consider a homogeneous ten-dimensional metric and a six-dimensional torus, $T^{6}$ as a compactified space. The scale factors are functions of the time coordinate. The $T^{6}$ has six scale factors corresponding to six cycles. The string-frame metric is given by the following equation:

$$
d s_{10}^{2}=-e^{2 \lambda_{0}(t)} d t^{2}+e^{2 \lambda(t)} d \mathbf{x}^{2}+\sum_{m=4}^{9} e^{2 \lambda_{m}(t)}\left(d y^{m}\right)^{2}
$$

where $d \mathbf{x}^{2} \equiv \sum_{i=1}^{3}\left(d x^{i}\right)^{2}$ is the line element of the flat three-dimensional space, $R^{3}$ or $T^{3}$ and the cycle of $T^{6}$ is defined as

$$
0 \leq y^{m} \leq 2 \pi \sqrt{\alpha^{\prime}} .
$$

$\alpha^{\prime}$ is related with a string length as $l_{\mathrm{s}} \sim \sqrt{\alpha^{\prime}}$. The scale factor $e^{\lambda_{m}(t)}$ describes the scaling of the cycle defined by (2). The four-dimensional Einstein frame is defined by the following transformation:

$$
\begin{aligned}
\lambda_{0}(t) & =n(t)+\beta(t), & \beta(t) & \equiv \phi(t)-\frac{1}{2} \bar{\lambda}(t), \\
\lambda(t) & =A(t)+\beta(t), & \bar{\lambda}(t) & \equiv \sum_{m=4}^{9} \lambda_{m}(t)
\end{aligned}
$$

where $\phi(t)$ is the dilaton field. $e^{n(t)}$ and $e^{A(t)}$ are the lapse function and the scale factor of the three-dimensional space, respectively. For the Einstein frame the proper time of the four-dimensional space-time is defined by $e^{n(t)}=1$ which is not equivalent to the proper time of the ten-dimensional space-time in the string frame because of $e^{\lambda_{0}(t)}=e^{\beta(t)}$.

By (3) the dilaton gravity sector of the string-frame action becomes [7] 


$$
\begin{aligned}
S_{0}= & \frac{1}{16 \pi G_{10}} \int_{M^{10}} d^{10} X \sqrt{-G} e^{-2 \phi(t)}\left(R+4(\nabla \phi)^{2}\right) \\
= & \frac{1}{16 \pi G_{4}} \int_{M^{4}} d^{4} x e^{n(t)+3 A(t)} \\
& \times e^{-2 n(t)}\left(-6 \dot{A}^{2}(t)+2 \dot{\beta}^{2}(t)+\sum_{m=4}^{9} \dot{\lambda}_{m}^{2}(t)\right)
\end{aligned}
$$

where the four-dimensional Newton constant and the tendimensional gravitational constant are given by [31]

$$
\begin{gathered}
G_{4}=\frac{G_{10}}{\left(2 \pi \sqrt{\alpha^{\prime}}\right)^{6}}=\frac{\alpha^{\prime}}{8}, \\
\kappa_{10}^{2}=8 \pi G_{10}=\frac{1}{2}(2 \pi)^{7} \alpha^{\prime 4} .
\end{gathered}
$$

Equation (4) shows that the four-dimensional EinsteinHilbert term which is given by the first term of the second line does not involve moduli fields and the dilaton. By (5) we find that the string length is of the order of the Planck length. An advantage of the new variable $\beta(t)$ is that the kinetic term of the above action becomes diagonal.

\section{POINT PARTICLES FROM WRAPPED BRANES}

In the previous section, we have defined the fourdimensional Einstein frame which gives the fourdimensional Einstein-Hilbert term with the timeindependent Newton constant and diagonal kinetic terms of moduli fields. We found that the dynamics of the moduli fields is governed by potential terms derived by flux terms and brane sources after the Kaluza-Klein reduction. In this section, we will consider the effective action of the wrapped D $p$-brane, NS5-brane, and KK5-monopole, using the four-dimensional Einstein frame and derive the energy density of those ingredients which contribute as potential terms for the moduli fields.

We consider branes wrapping over only cycles of $T^{6}$. After the compactification, we see those objects as point particles on the three-dimensional space. This expectation is motivated by Ref. [25] which shows that wrapped brane gases give the energy density of a pressureless matter. In [25], we gave the effective action without a dependence of the velocity along the three-dimensional space. We would like to derive the effective action with the velocity on the three-dimensional space. The effective action will be used to read off the mass and the charge of a wrapped brane in later sections. To derive the effective action we assume a cancellation of total RR charges of the D-branes, because of homogeneous distributions of many D-branes on the nine-dimensional space by the brane gas approximation. Thus, we will assume wrapped branes as gases of free particles.

First, we will consider the world volume action of $\mathrm{D} p$-brane wrapping over a $p$-dimensional cycle of $T^{6}$. If the $\mathrm{D} p$-brane wraps over a $\left(m_{1}, \ldots, m_{p}\right)$ cycle $(0 \leq p \leq$
6), gauge fields on the $\mathrm{D} p$-brane exist along the $\left(m_{1}, \ldots, m_{p}\right)$ directions. The $\mathrm{D} p$-brane can move along transverse directions. We assume that the distributions of the branes are homogeneous and that the gauge field is Abelian. Then the gauge potential and transverse coordinates depend only on the time variable, i.e., $A_{m_{a}}(t), X^{i}(t)$ $(i=1,2,3)$, and $X^{m_{a}}(t)$. We will adopt the coordinate system as $\xi^{0}=t, \xi^{m_{a}}=y^{m_{a}}(a=1,2, \ldots, p)$. Then, the Dirac-Born-Infeld action of $\mathrm{D} p$-brane wrapping over the $\left(m_{1}, \ldots, m_{p}\right)$ cycle, $\Sigma_{p}$ is given by

$$
\begin{aligned}
S_{\mathrm{D} p}^{\left(m_{1} \cdots m_{p}\right)}= & -T_{p} \int_{R \times \Sigma_{p}} d^{p+1} \xi e^{-\phi(t)} \sqrt{-\operatorname{det}\left(\gamma_{a b}+2 \pi \alpha^{\prime} F_{a b}\right)} \\
= & -\left(2 \pi \sqrt{\alpha^{\prime}}\right)^{p} T_{p} \int d t e^{-\phi(t)+\lambda_{0}(t)+\sum_{a=1}^{p} \lambda_{m_{a}}(t)} \\
& \times\left\{1-e^{-2 \lambda_{0}(t)+2 \lambda(t)} \sum_{i=1}^{3}\left(\dot{X}^{i}(t)\right)^{2}-\left(2 \pi \alpha^{\prime}\right)^{2}\right. \\
& \times \sum_{a=1}^{p} e^{-2 \lambda_{0}(t)-2 \lambda_{m_{a}}(t)}\left(\dot{A}_{m_{a}}(t)\right)^{2} \\
& \left.-\sum_{b=p+1}^{6} e^{-2 \lambda_{0}(t)+2 \lambda_{m_{b}}(t)}\left(\dot{X}^{m_{b}}(t)\right)^{2}\right\}^{1 / 2}
\end{aligned}
$$

where the tension of the $\mathrm{D} p$-brane is given by [31]

$$
T_{p}=(2 \pi)^{-p}\left(\alpha^{\prime}\right)^{-(p+1) / 2} .
$$

$\sum_{i=1}^{3}\left(\dot{X}^{i}(t)\right)^{2}$ denotes the velocity along the threedimensional space. The world volume action is proportional to $\exp (-\phi) \sqrt{\operatorname{det} \gamma_{a b}}$ and then the mass becomes heavy for $e^{\phi} \ll 1$ and $e^{\lambda_{m}} \gg 1$.

The above action is defined in the string frame. We have to take the four-dimensional frame to extract the fourdimensional dynamics. Substituting (3) for (7), we obtain

$$
\begin{aligned}
S_{\mathrm{D} p}^{\left(m_{1} \cdots m_{p}\right)}= & -\left(2 \pi \sqrt{\alpha^{\prime}}\right)^{p} T_{p} \int d t e^{n(t)-(1 / 2) \bar{\lambda}(t)+\sum_{a=1}^{p} \lambda_{m_{a}}(t)} \\
& \times\left\{1-e^{-2 n(t)+2 A(t)} \sum_{i=1}^{3}\left(\dot{X}^{i}(t)\right)^{2}-\mathcal{A}(t)\right\}^{1 / 2}
\end{aligned}
$$

where $\mathcal{A}(t)$ is defined by the following equation:

$$
\begin{aligned}
\mathcal{A}(t) \equiv & \left(2 \pi \alpha^{\prime}\right)^{2} \sum_{a=1}^{p} e^{-2 \beta(t)-2 n(t)-2 \lambda_{m_{a}}(t)}\left(\dot{A}_{m_{a}}(t)\right)^{2} \\
& +\sum_{b=p+1}^{6} e^{-2 \beta(t)-2 n(t)+2 \lambda_{m_{b}}(t)}\left(\dot{X}^{m_{b}}(t)\right)^{2} .
\end{aligned}
$$

Compared with (9) to (7), we find the basic difference between the string frame and Einstein frame. In the string frame, the winding mode of the world volume depends on the dilaton, whereas the winding mode of the $\mathrm{D} p$-brane does not have the coupling of the dilaton field in the fourdimensional Einstein frame. This fact implies that the winding mode cannot stabilize the dilaton field in the 
four-dimensional Einstein frame. Namely, if we take $\mathcal{A}(t)$ defined by (10), we also cannot stabilize the dilaton, since $\mathcal{A}(t)$ includes the dilaton with $e^{-2 \beta(t)}$ only. Therefore, the $\mathrm{D} p$-brane wrapping over cycles of $T^{6}$ cannot stabilize the dilaton field in the four-dimensional Einstein frame, using the cosmological background defined by (1).

The wrapped branes can be regarded as the ideal gas based on the dilute gas approximation. We can solve the equations of motion of $A_{m_{a}}(t)$ and $X^{m_{b}}(t)$ derived from (9) as follows:

$$
\begin{gathered}
e^{n(t)-(1 / 2) \bar{\lambda}(t)+\sum_{a^{\prime}=1}^{p} \lambda_{m_{a^{\prime}}}(t)}\left(2 \pi \alpha^{\prime}\right) e^{-2 \beta(t)-2 n(t)-2 \lambda_{m_{a}}(t)} \dot{A}_{m_{a}}(t) \\
=\frac{f_{m_{a}}}{2 \pi \alpha^{\prime}}\left\{1-e^{-2 n(t)+2 A(t)} \sum_{i=1}^{3}\left(\dot{X}^{i}(t)\right)^{2}-\mathcal{A}(t)\right\}^{1 / 2},
\end{gathered}
$$

$$
\begin{gathered}
e^{n(t)-(1 / 2) \bar{\lambda}(t)+\sum_{a^{\prime}=1}^{p} \lambda_{m_{a^{\prime}}}(t)} e^{-2 \beta(t)-2 n(t)+2 \lambda_{m_{b}}(t)} \dot{X}^{m_{b}}(t) \\
=v^{m_{b}}\left\{1-e^{-2 n(t)+2 A(t)} \sum_{i=1}^{3}\left(\dot{X}^{i}(t)\right)^{2}-\mathcal{A}(t)\right\}^{1 / 2}
\end{gathered}
$$

where we have the equations of motions and $f_{m_{a}}$ and $v^{m_{b}}$ are constants of integration. $f_{m_{a}}$ and $v^{m_{b}}$ are integers and not continuous numbers, because of a cyclicity of the $T^{6}$. For example, $v^{m_{b}}$ is related to a conjugate momentum $P_{\mathrm{D} p, m_{b}}^{m_{1} \cdots m_{p}} \equiv \partial \mathcal{L}_{\mathrm{D} p}^{m_{1} \cdots m_{m_{p}}} / \partial \dot{X}^{m_{b}}$ as $P_{\mathrm{D} p, m_{b}}^{m_{1} \cdots m_{p}}=v^{m_{b}} / \sqrt{\alpha^{\prime}}$ by (12). The conjugate momentum must be an integer on a cycle of the $T^{6}$ therefore $v^{m_{b}}$ is an integer. By the $T$ dulaity $f_{m_{a}}$ and $v^{m_{b}}$ are mapped to each other [25] and then $f_{m_{a}}$ must also be an integer. The similar property on a quantized momentum is satisfied for a fundamental string as shown in Appendix C.

We can solve Eqs. (11) and (12) on $\mathcal{A}(t)$ :

$$
\begin{aligned}
\mathcal{A}(t)= & \frac{e^{\bar{\lambda}-2 \sum_{a^{\prime}=1}^{p} \lambda_{m_{a^{\prime}}}(t)} \tilde{\mathcal{A}}(t)}{1+e^{\bar{\lambda}-2 \sum_{a^{\prime}=1}^{p} \lambda_{m_{a^{\prime}}}(t)} \tilde{\mathcal{A}}(t)} \\
& \times\left\{1-e^{-2 n(t)+2 A(t)} \sum_{i=1}^{3}\left(\dot{X}^{i}(t)\right)^{2}\right\}, \\
\tilde{\mathcal{A}}(t) \equiv & \sum_{a=1}^{p} e^{2 \beta(t)+2 \lambda_{m_{a}}(t)}\left(\frac{f_{m_{a}}}{2 \pi \alpha^{\prime}}\right)^{2} \\
& +\sum_{b=p+1}^{6} e^{2 \beta(t)-2 \lambda_{m_{b}}(t)}\left(v^{m_{b}}\right)^{2} .
\end{aligned}
$$

In the four-dimensional Einstein frame, the potential term is derived by

$$
\begin{aligned}
u_{\mathrm{D} p}^{\left(m_{1} \cdots m_{p}\right)} & \equiv-T_{0}^{0}=\frac{2 g^{00}}{\sqrt{-g_{4}(x)}} \frac{\delta \mathcal{L}_{\mathrm{D} p}^{\left(m_{1} \cdots m_{p}\right)}}{\delta g^{00}} \\
& =-e^{-n(t)-3 A(t)} \frac{\delta \mathcal{L}_{\mathrm{D} p}^{\left(m_{1} \cdots m_{p}\right)}}{\delta n(t)} .
\end{aligned}
$$

Using (9), (13), and (14), we obtain the potential of the $\mathrm{D} p$-brane wrapping over the $\left(m_{1} \cdots m_{p}\right)$ cycle:

$$
\begin{aligned}
u_{\mathrm{D} p}^{\left(m_{1} \cdots m_{p}\right)}= & e^{-3 A(t)}\left(2 \pi \sqrt{\alpha^{\prime}}\right)^{p} T_{p} \times\left\{e^{-\bar{\lambda}(t)+2 \sum_{a=1}^{p} \lambda_{m_{a}}(t)}\right. \\
& +\tilde{\mathcal{A}}(t)\}^{1 / 2} \times\left\{1-e^{-2 n(t)+2 A(t)} \sum_{i=1}^{3}\left(\dot{X}^{i}(t)\right)^{2}\right\}^{-(1 / 2)} .
\end{aligned}
$$

This corresponds to the energy density of a relativistic particle with the mass given by

$$
\begin{aligned}
m_{p}^{\left(m_{1} \cdots m_{p}\right)}= & \left(2 \pi \sqrt{\alpha^{\prime}}\right)^{p} T_{p} \times\left\{e^{-\bar{\lambda}(t)+2 \sum_{a=1}^{p} \lambda_{m_{a}}(t)}\right. \\
& +\tilde{\mathcal{A}}(t)\}^{1 / 2} .
\end{aligned}
$$

Thus, we see $\mathrm{D} p$-branes behave as point particles on the three-dimensional space as Fig. 1. The world volume decays with $e^{-3 A(t)}$ as the pressureless matter.

The mass given by (16) has the following role as a potential of the moduli fields. The winding part of (16) is proportional to

$$
\exp \frac{1}{2}\left(+\sum_{a=1}^{p} \lambda_{m_{a}}(t)-\sum_{b=p+1}^{6} \lambda_{m_{b}}(t)\right)
$$

This indicates that the wrapped $\mathrm{D} p$-brane binds the $\left(m_{1} \cdots m_{p}\right)$ cycle where the $\mathrm{D} p$-brane expands and the $\left(m_{p+1} \cdots m_{6}\right)$ direction is stretched like a rubber band as in Fig. 2. Similarly, by (13) it is found that the electric fields in $\tilde{\mathcal{A}}(t)$ also bind the $\left(m_{1} \cdots m_{p}\right)$ cycle. The transverse velocities in $\tilde{\mathcal{A}}(t)$ make a pressure bringing the expansion to the transverse directions.

We will derive the action of the point particle for the NS5-brane and KK5-monopole in type II string theory which has five spatial dimensions. In this paper, we consider only winding modes of the NS5-brane and KK5monopole moving along the three-dimensional space. The world volume action of the KK5-monopole wrapping over the $\left(m_{1} \cdots m_{5}\right)$ cycle is given by $[32,33]$

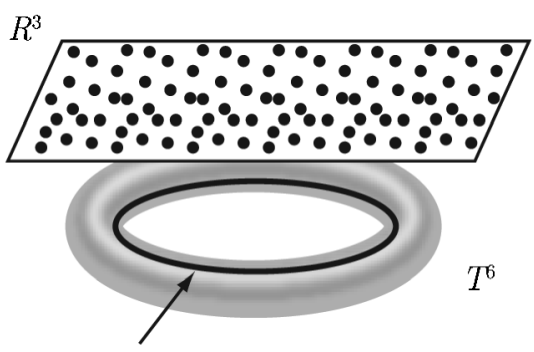

Wrapped branes

FIG. 1. Wrapped brane gases can be seen as point particles on three dimensions. Those point particles decay as the pressureless dust with $e^{-3 A(t)}$. 


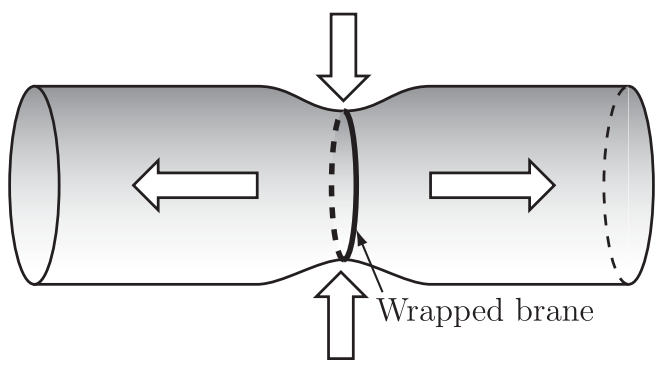

FIG. 2. A cycle where branes wrap is bound by the tension of branes. Cycles where branes do not wrap are stretched like a rubber tube in the four-dimensional Einstein frame. The cycles are homogeneously bound under the brane gas approximation, although this figure shows one of branes as an example.

$$
\begin{aligned}
S_{\mathrm{KK} 5}^{\left(m_{1} \cdots m_{5}\right)}= & -T_{\mathrm{KK} 5} \int_{R \times \Sigma_{5}} d^{6} \xi e^{-2 \phi(t)} k^{2} \sqrt{-\operatorname{det} \tilde{\gamma}_{a b}} \\
= & -\left(2 \pi \sqrt{\alpha^{\prime}}\right)^{5} T_{\mathrm{KK} 5} \\
& \times \int d t e^{-2 \phi(t)+2 \lambda_{6}(t)+\lambda_{0}(t)+\sum_{a=1}^{5} \lambda_{m_{a}}(t)} \\
& \times\left\{1-e^{-2 \lambda_{0}(t)+2 \lambda(t)} \sum_{i=1}^{3}\left(\dot{X}^{i}(t)\right)^{2}\right\}^{1 / 2}, \\
\tilde{\gamma}_{a b} \equiv & \frac{\partial X^{m}}{\partial \xi^{a}} \frac{\partial X^{m}}{\partial \xi^{a}}\left(G_{m n}-k^{-2} k_{m} k_{n}\right),
\end{aligned}
$$

where $k^{m} \equiv \delta^{m m_{6}}$ is the killing vector of the $S^{1}$ isometry along the $\left(m_{6}\right)$ cycle and $k^{2} \equiv G_{m n} k^{m} k^{n}=\exp \left(2 \lambda_{m_{6}}(t)\right)$. We also consider the NS5-brane. The world volume action of the NS5-brane [34] wrapping over $\left(m_{1} \cdots m_{5}\right)$ cycles is given by

$$
\begin{aligned}
S_{\mathrm{NS} 5}^{\left(m_{1} \cdots m_{5}\right)}= & -T_{\mathrm{NS} 5} \int_{R \times \Sigma_{5}} d^{6} \xi e^{-2 \phi(t)} \sqrt{-\operatorname{det} \gamma_{a b}} \\
= & -\left(2 \pi \sqrt{\alpha^{\prime}}\right)^{5} T_{\mathrm{NS} 5} \int d t e^{-2 \phi(t)+\lambda_{0}(t)+\sum_{a=1}^{5} \lambda_{m_{a}}(t)} \\
& \times\left\{1-e^{-2 \lambda_{0}(t)+2 \lambda(t)} \sum_{i=1}^{3}\left(\dot{X}^{i}(t)\right)^{2}\right\}^{1 / 2}
\end{aligned}
$$

We consider the transformation of fields as in (3) to obtain the potential term of the NS5-brane and the KK5monopole in the four-dimensional Einstein frame. Substituting (3) for (17) and (18) and replacing $\mathcal{L}_{\mathrm{D} p}^{\left(m_{1} \cdots m_{p}\right)}$ with $\mathcal{L}_{\mathrm{NS} 5 / \mathrm{KK} 5}^{\left(m_{1} \cdots m_{5}\right)}$ in (14), the energy density of the NS5-brane and the KK5-monopole are given by

$$
\begin{aligned}
u_{\mathrm{NS} 5}^{\left(m_{1} \cdots m_{5}\right)}= & e^{-3 A(t)}\left(2 \pi \sqrt{\alpha^{\prime}}\right)^{5} T_{\mathrm{NS} 5} e^{-\beta(t)-\bar{\lambda}(t)+\sum_{a=1}^{5} \lambda_{m_{a}}(t)} \\
& \times\left\{1-e^{-2 n(t)+2 A(t)} \sum_{i=1}^{3}\left(\dot{X}^{i}(t)\right)^{2}\right\}^{-(1 / 2)}
\end{aligned}
$$

$$
\begin{aligned}
u_{\mathrm{KK} 5}^{\left(m_{1} \cdots m_{5}\right)}= & e^{-3 A(t)}\left(2 \pi \sqrt{\alpha^{\prime}}\right)^{5} T_{\mathrm{KK} 5} e^{-\beta(t)-\bar{\lambda}(t)+2 \lambda_{m_{6}}+\sum_{a=1}^{5} \lambda_{m_{a}}(t)} \\
& \times\left\{1-e^{-2 n(t)+2 A(t)} \sum_{i=1}^{3}\left(\dot{X}^{i}(t)\right)^{2}\right\}^{-(1 / 2)},
\end{aligned}
$$

where $T_{\mathrm{D} 5}=T_{\mathrm{NS} 5}=T_{\mathrm{KK} 5}$ [33]. The mass of the particles is given by

$$
\begin{aligned}
& m_{\mathrm{NS} 5}^{\left(m_{1} \cdots m_{5}\right)}=\left(2 \pi \sqrt{\alpha^{\prime}}\right)^{5} T_{\mathrm{NS} 5} \times e^{-\beta(t)-\lambda_{m_{6}}(t)}, \\
& m_{\mathrm{KK} 5}^{\left(m_{1} \cdots m_{5}\right)}=\left(2 \pi \sqrt{\alpha^{\prime}}\right)^{5} T_{\mathrm{KK} 5} \times e^{-\beta(t)+\lambda_{m_{6}}(t)} .
\end{aligned}
$$

It is expected that the world volume of NS5 and KK5 contributes to the stabilization of the dilaton, since those objects have the opposite dependence on the dilaton, compared with (16). In fact, in [25] the authors have suggested that a D1-KK5 brane gas model stabilizes the dilaton field as well as the radial moduli fields, simultaneously.

\section{MASS OF WRAPPED BRANES IN THE FOUR- DIMENSIONAL EINSTEIN FRAME}

In Sec. III, the effective mass of the wrapped brane is derived in the four-dimensional Einstein frame. In this section, we would like to consider a behavior of the mass derived in the previous section, taking a large scale of the $T^{6}$ and a weak string coupling related with the dilaton field as $g_{s}=\exp (\phi)$. The condition is required to consider an effective field theory. It is not necessarily correct that the $\mathrm{D} p$-brane is very heavy with a large volume and a weak string coupling, since the world volume action has the nontrivial coupling to the dilaton and the scale of $T^{6}$ in the four-dimensional Einstein frame. We take the string scale as $1 / \sqrt{\alpha^{\prime}} \sim m_{\text {Planck }} \sim \mathcal{O}\left(10^{19}\right) \mathrm{GeV}$ to analyze the mass.

First, we will consider winding modes of branes and $e^{\lambda_{m}(t)}=e^{\lambda^{\prime}(t)}$. By (16), (21), and (22) the mass of branes is as follows:

$$
\begin{aligned}
m_{p, \text { winding }}^{\left(m_{1} \cdots m_{p}\right)} & =\frac{1}{\sqrt{\alpha^{\prime}}} \times e^{(p-3) \lambda^{\prime}(t),} \\
m_{\mathrm{NS} 5, \text { winding }}^{\left(m_{1} \cdots m_{5}\right)} & =\frac{1}{\sqrt{\alpha^{\prime}}} \times e^{-\phi(t)+2 \lambda^{\prime}(t)}, \\
m_{\mathrm{KK} 5, \text { winding }}^{\left(m_{1} \cdots m_{5}\right)} & =\frac{1}{\sqrt{\alpha^{\prime}}} \times e^{-\phi(t)+4 \lambda^{\prime}(t)} .
\end{aligned}
$$

We find that the mass of NS5 and KK5 becomes very heavy for $e^{\lambda^{\prime}} \gg 1$ and $e^{\phi} \ll 1$. In the case of $p \geq 4$, the mass of the $\mathrm{D} p$-brane also becomes heavy. The D3-brane has the Planck mass. On the other hand, for $p \leq 2$, the $\mathrm{D} p$-brane has the light mass under the condition of $e^{\lambda^{\prime}} \gg 1$ and $e^{\phi} \ll 1$. For example, we will consider $\exp \left(\lambda^{\prime}\right) \sim \mathcal{O}\left(10^{5}\right)$ which implies that the compactification is around the grand unified theory scale,

$$
V_{T^{6}}=\left(2 \pi \sqrt{\alpha^{\prime}} \exp \left(\lambda^{\prime}\right)\right)^{6} \sim\left(\mathcal{O}\left(10^{-14}\right) \mathrm{GeV}^{-1}\right)^{6} .
$$


The mass of the $\mathrm{D} p$-brane is of the order of

$$
m_{p \text {,winding }}^{\left(m_{1} \cdots m_{p}\right)} \sim \mathcal{O}\left(10^{5(p-3)+19}\right) \mathrm{GeV} .
$$

The mass ranges from $\mathcal{O}(10) \mathrm{TeV}$ for $p=0$ to $\mathcal{O}\left(10^{14}\right) \mathrm{GeV}$ for $p=2$. This is the nontrivial result, since the string-frame gives rise to heavy branes for the large volume. We should include those light states in string cosmologies. Taking the four-dimensional Einstein frame gives the interesting result for the mass of wrapped Dbranes.

Second, we will consider the anisotropic case, $\lambda_{m} \neq \lambda_{n}$ satisfying $\exp \left(\lambda_{m}\right) \gg 1$ for the large volume condition. By (16) the masses of the wrapped $\mathrm{D} p$ - and $\mathrm{D}(6-p)$-brane can be represented as

$$
\begin{aligned}
m_{p, \text { winding }}^{\left(m_{1} \cdots m_{p}\right)} & =\frac{1}{\sqrt{\alpha^{\prime}}} \exp \left(\sum_{a=1}^{p} \frac{\lambda_{m_{a}}(t)}{2}-\sum_{b=p+1}^{6} \frac{\lambda_{m_{b}}(t)}{2}\right), \\
m_{6-p, \text { winding }}^{\left(m_{p+1} \cdots m_{6}\right)} & =\frac{1}{\sqrt{\alpha^{\prime}}} \exp \left(\sum_{a=p+1}^{6} \frac{\lambda_{m_{a}}(t)}{2}-\sum_{b=1}^{p} \frac{\lambda_{m_{b}}(t)}{2}\right) .
\end{aligned}
$$

Those masses explicitly satisfy the following electricmagnetic dual relation:

$$
m_{p, \text { winding }}^{\left(m_{1} \cdots m_{p}\right)} \times m_{6-p, \text { winding }}^{\left(m_{p+1} \cdots m_{6}\right)}=\left(\frac{1}{\sqrt{\alpha^{\prime}}}\right)^{2} .
$$

The right-hand side of the above equation is independent on scale factors, while masses depend on the time. Thus, masses of the $\mathrm{D} p$ - and $\mathrm{D}(6-p)$-branes are not independent of each other and satisfy a seesaw like condition. A $\mathrm{D} p$-brane becomes light when a $\mathrm{D}(6-p)$-brane has a heavy mass and vice versa. The electric-magnetic dual relation is one of the reasons for this scaling property. The behavior of the mass can be classified into three parts by a dynamics of the moduli fields as follows.

(1) $\sum_{a=1}^{p} \lambda_{m_{a}}(t)-\sum_{b=p+1}^{6} \lambda_{m_{b}}(t)>0$ : the mass of the $\mathrm{D} p$-brane is heavy and $\mathrm{D}(6-p)$ becomes light.

(2) $\sum_{a=1}^{p} \lambda_{m_{a}}(t)-\sum_{b=p+1}^{6} \lambda_{m_{b}}(t)=0$ : the masses of $\mathrm{D} p$ - and $\mathrm{D}(6-p)$-branes are of order $\mathcal{O}\left(1 / \sqrt{\alpha^{\prime}}\right) \sim$ $\mathcal{O}\left(m_{\text {Planck }}\right)$.

(3) $\sum_{a=1}^{p} \lambda_{m_{a}}(t)-\sum_{b=p+1}^{6} \lambda_{m_{b}}(t)<0$ : the mass of the $\mathrm{D}(6-p)$-brane is heavy and the $\mathrm{D} p$-brane becomes light.

A comment is in order about the dimensionality of space-times. The time-independent electric-magnetic dual relation (26) is realized only in the four-dimensional Einstein frame. For example, the mass of the wrapped $\mathrm{D} p$-brane in the string frame is given by

$$
m_{p, \text { winding }}^{\left(\text {string } \mid m_{1} \cdots m_{p}\right)}=\frac{1}{\sqrt{\alpha^{\prime}}} e^{-\phi(t)+\lambda_{m_{1}}(t)+\cdots+\lambda_{m_{p}}(t)}
$$

and the dual relation is [35]

$$
\begin{aligned}
& m_{p, \text { winding }}^{\left(\text {string } \mid m_{1} \cdots m_{p}\right)} \times m_{6-p, \text { winding }}^{\left(\text {string } \mid m_{p+1} \cdots m_{6}\right)} \\
& \quad=\left(\frac{1}{\sqrt{\alpha^{\prime}}}\right)^{2} \exp (-2 \phi(t)+\bar{\lambda}(t)) .
\end{aligned}
$$

This relation explicitly depends on time. If the right-hand side of (27) becomes large, each mass of wrapped branes can take a large value. Similarly, using the $(d+1)$ dimensional Einstein frame given by (A2), the mass of the wrapped $\mathrm{D} p$-brane is given by

$$
\begin{aligned}
m_{p, \text { winding }}^{\left(d+1 \mid m_{1} \cdots m_{p}\right)}= & \frac{1}{\sqrt{\alpha^{\prime}}} \exp \left(\frac{3-d}{d-1} \phi(t)+\sum_{a=1}^{p} \lambda_{m_{a}}(t)\right. \\
& \left.-\frac{1}{d-1} \sum_{m=d+1}^{9} \lambda_{m}(t)\right)
\end{aligned}
$$

and the dual relation is

$$
\begin{aligned}
& m_{p, \text { winding }}^{\left(d+1 \mid m_{1} \cdots m_{p}\right)} \times m_{10-(d+1)-p \text {, winding }}^{\left(d+1 \mid m_{p+1} \cdots m_{10-(d+1)}\right)} \\
& \quad=\left(\frac{1}{\sqrt{\alpha^{\prime}}}\right)^{2} \exp \frac{d-3}{d-1}\left(-2 \phi(t)+\sum_{m=d+1}^{9} \lambda_{m}(t)\right) .
\end{aligned}
$$

The right-hand side also involves time-dependent scale factors. Therefore, the four-dimensional Einstein frame is a special case where the electric-magnetic dual relation becomes scale-free.

By the $S$ - and $T$-duality, it is expected that the mass of the wrapped NS5-brane and KK5-monopole has a dual relation as (26). Using $S$-dual rule in the four-dimensional Einstein frame (B3) and (B4), the winding modes of D1and D5-branes are mapped to the winding modes of fundamental string given by the first term of (C5) with $w^{m_{a}}=$ 1 and the NS5-brane, respectively. Then wrapped fundamental string and NS5-brane satisfy the following dual relation:

$$
m_{\mathrm{F} 1 \text {,winding }}^{\left(m_{1}\right)} \times m_{\mathrm{NS} 5}^{\left(m_{2} \cdots m_{6}\right)}=\left(\frac{1}{\sqrt{\alpha^{\prime}}}\right)^{2}
$$

where we have used (21) and (C5). According to $T$-dual along the $\left(m_{1}\right)$-cycle $\left(\lambda_{m_{1}} \rightarrow-\lambda_{m_{1}}, \phi \rightarrow \phi-\lambda_{m_{1}}\right)$, the mass of the wrapped NS5-brane is mapped to the mass of the KK5-monopole as $m_{\mathrm{NS} 5}^{\left(m_{2} \cdots m_{6}\right)} \rightarrow m_{\mathrm{KK} 5}^{\left(m_{2} \cdots m_{6}\right)}$ [25]. According to $T$-dual along the $\left(m_{1}\right)$-cycle $\left(\lambda_{m_{1}} \rightarrow-\lambda_{m_{1}}\right.$, $\left.\phi \rightarrow \phi-\lambda_{m_{1}}\right)$, the mass of the wrapped NS5-brane is mapped to the mass of the KK5-monopole as $m_{\mathrm{NS} 5}^{\left(m_{2} \cdots m_{6}\right)} \rightarrow$ $m_{\mathrm{KK} 5}^{\left(m_{2} \cdots m_{6}\right)}$ [25]. The winding mode of the fundamental string is mapped to the momentum mode as $m_{\mathrm{F} 1 \text {,winding }}^{\left(m_{1}\right)} \rightarrow$ $m_{\mathrm{F} 1 \text {,momentum }}^{\left(m_{1}\right)}=\frac{1}{\sqrt{\alpha^{\prime}}} e^{\beta(t)-\lambda_{m_{1}}(t)}, \quad\left(n_{m_{1}}=1\right)$. Therefore we obtain

$$
m_{\mathrm{F} 1, \text { momentum }}^{\left(m_{1}\right)} \times m_{\mathrm{KK} 5}^{\left(m_{2} \cdots m_{6}\right)}=\left(\frac{1}{\sqrt{\alpha^{\prime}}}\right)^{2} .
$$

The right-hand side of (30) and (31) is scale-free in the 
four-dimensional Einstein frame. For example, if $m_{\mathrm{F} 1 \text {,winding }}^{\left(m_{1}\right)}$ and $m_{\mathrm{F} 1 \text {,momentum }}^{\left(m_{1}\right)}$ are lighter than the Planck mass, the mass of the wrapped NS5-brane and the wrapped KK5-monopole becomes very heavy.

The dual relations (30) and (31) are related with the stabilization of the dilaton field. The winding and the momentum mode of the fundamental string is proportional to $e^{+\beta}$. By dual relations, the mass of NS5 and KK5 must have the dependence of $e^{-\beta}$ on the dilaton field. NS5 and KK5 are essential for the stabilization of the dilaton [25]. Thus the dual relation of wrapped branes plays an important role for the moduli stabilization.

\section{MASS OF WRAPPED BRANES WITH CALABI-YAU THREEFOLD}

In the previous section, we have found that there are cases where the mass of the brane wrapping over cycles of the $T^{6}$ becomes light in the four-dimensional Einstein frame. One may expect light wrapped branes for the $\mathrm{CY}_{3}$ compactification. In this section, we will investigate masses of $\mathrm{D} p$-branes wrapping over cycles of the $\mathrm{CY}_{3}$, taking the four-dimensional Einstein frame. To analyze the mass we control scales of the Calabi-Yau by hand because we cannot show the moduli stabilization of the Calabi-Yau in this paper. It is also interesting to know whether masses satisfy the electric-magnetic dual relation found in the previous section.

The $\mathrm{CY}_{3}$ has real six dimensions, 2, 3, and 4 cycles. D0-, D2-, D3-, D4-, and D6-brane can live in the $\mathrm{CY}_{3}$, while D1-, D5-, NS5-brane, and KK5-monopole cannot wrap a cycle of the $\mathrm{CY}_{3}$. In this section, we assume a large volume (complex structure) limit and ignore any quantum correction. We do not consider instabilities of wrapped branes and assume the supersymmetric cycle condition behaves well. To obtain the four-dimensional Einstein frame with a $\mathrm{CY}_{3}$ compactification we redefine $\beta(t)$ as

$$
\beta(t)=\phi(t)-\frac{1}{2} \ln \left(\frac{V_{\mathrm{CY}_{3}}(t)}{\left(2 \pi \sqrt{\alpha^{\prime}}\right)^{6}}\right)
$$

where we have defined $V_{\mathrm{CY}_{3}}(t) \equiv \int d^{6} y \sqrt{g_{\mathrm{CY}_{3}}(t)}$. The volume of the $\mathrm{CY}_{3}$ has a time dependence through moduli fields.

If $\mathrm{D} p$-branes $(p=0,2,4,6)$ are included minimally in the $\mathrm{CY}_{3}$ or its cycle, we can represent winding modes of the $\mathrm{D} p$-branes as

$$
\begin{aligned}
m_{0} & =\left(2 \pi \sqrt{\alpha^{\prime}}\right)^{3} T_{0} V_{\mathrm{CY}_{3}}^{-1 / 2}, \\
m_{2}^{(A)} & =\left(2 \pi \sqrt{\alpha^{\prime}}\right)^{3} T_{2} V_{\mathrm{CY}_{3}}^{-1 / 2} \times \int_{\Sigma_{2}^{A}} J, \\
m_{4,(A)} & =\left(2 \pi \sqrt{\alpha^{\prime}}\right)^{3} T_{4} V_{\mathrm{CY}_{3}}^{-1 / 2} \times \frac{1}{2} \int_{\Sigma_{4, A}} J \wedge J, \\
m_{6} & =\left(2 \pi \sqrt{\alpha^{\prime}}\right)^{3} T_{6} V_{\mathrm{CY}_{3}}^{1 / 2},
\end{aligned}
$$

where $J$ is the Kähler form, $\Sigma_{2}^{A}$ and $\Sigma_{4, A}$ are dual to the harmonic form $\omega_{A}$ and $\tilde{\omega}^{A}$. The properties of the Kähler form are explained in Appendix D. The Poincaré dual gives rise to

$$
\begin{gathered}
\int_{\Sigma_{2}^{A}} J=\int_{\mathrm{CY}_{3}} J \wedge \tilde{\omega}^{A}=v^{A}, \\
\int_{\Sigma_{4, A}} J \wedge J=\int_{\mathrm{CY}_{3}} J \wedge J \wedge \omega_{A} \equiv \mathcal{K}_{A B C} v^{B} v^{C} .
\end{gathered}
$$

Using the above equations and (D2), we obtain the following relations between masses of wrapped branes:

$$
m_{0} \times m_{6}=\left(\frac{1}{\sqrt{\alpha^{\prime}}}\right)^{2}, \quad \sum_{A \in h^{1,1}} m_{2}^{(A)} \times m_{4,(A)}=3\left(\frac{1}{\sqrt{\alpha^{\prime}}}\right)^{2} .
$$

Equation (34) implies that $m_{0}\left(m_{2}^{(A)}\right)$ is not independent of $m_{6}\left(m_{4,(A)}\right)$. In fact, by (D4) and (D5), there are relations between those masses as follows:

$$
\begin{aligned}
m_{6} & =-\left(2 \pi \sqrt{\alpha^{\prime}}\right)^{-6} \operatorname{Im} \mathcal{N}_{00} m_{0}, \\
m_{4,(A)} & =-\left(2 \pi \sqrt{\alpha^{\prime}}\right)^{-2} \operatorname{Im} \mathcal{N}_{A B} m_{2}^{(B)} .
\end{aligned}
$$

Note that the dual relation for D0- and D6-branes is satisfied quite naturally, since the dual relation does not require details of the $\mathrm{CY}_{3}$. For instance, a large $\mathrm{CY}_{3}$ with a condition as $V_{\mathrm{CY}_{3}} \sim\left(\mathcal{O}\left(10^{5}\right) \times 2 \pi \sqrt{\alpha^{\prime}}\right)^{6}$ gives $m_{0} \sim$ $\mathcal{O}(10) \mathrm{TeV}$. We consider a large volume by a constant scaling

$$
v^{A} \rightarrow \exp (2 s) v^{A}
$$

for all cycles. The $m_{2}^{(A)}$ and $m_{4,(A)}$ have the following scalings:

$$
\begin{aligned}
m_{2}^{(A)} & \rightarrow\left(2 \pi \sqrt{\alpha^{\prime}}\right)^{3} T_{2} V_{\mathrm{CY}_{3}}^{-1 / 2} \times e^{-s} \boldsymbol{v}^{A}, \\
m_{4,(A)} & \rightarrow\left(2 \pi \sqrt{\alpha^{\prime}}\right)^{3} T_{4} V_{\mathrm{CY}_{3}}^{-1 / 2} \times e^{+s} \mathcal{K}_{A B C} v^{B} v^{C}
\end{aligned}
$$

where $V_{\mathrm{CY}_{3}} \rightarrow \exp (+6 s) V_{\mathrm{CY}_{3}}$. Then light D2-branes and heavy D4-branes are realized for a positive constant $s$. If $\exp (2 s) \boldsymbol{v}^{A}$ is chosen as $\mathcal{O}\left(10^{10}\right) \times\left(2 \pi \sqrt{\alpha^{\prime}}\right)^{2}$, the $m_{2}^{(A)}$ is of order $\mathcal{O}\left(10^{15}\right) \mathrm{GeV}$. Those results have the same scalings given by (23) and (24).

The behavior of the D2- and D4-branes is more complicated than the D0-D6 case, because the dual relation for D2- and D4-branes cannot restrict all degrees of freedom of moduli fields. If we choose a scaling of $v^{A} \rightarrow \exp \left(2 s_{A}\right) v^{A}$, the masses of D2- and D4-branes vary correspondingly. Using (34) and (35), tuning the scale of cycles of the $\mathrm{CY}_{3}$, we can find models having light and heavy D2- and D4branes.

Quite similarly, we can consider a D3-brane wrapping over a 3 cycle. To derive the volume of the 3 cycle from $\mathrm{CY}_{3}$ data we will use a property of the special Lagrangian submanifold (supersymmetric cycle) [36-38]. Here, we 
assume that the condition of the special Lagrangian submanifold behaves well.

In general we call $N$ a special Lagrangian with a constant phase $\exp (i \theta)$, if $[36,38]$

$$
\begin{gathered}
\left.J\right|_{N}=0,\left.\quad[\sin \theta \operatorname{Re} \Omega-\cos \theta \operatorname{Im} \Omega]\right|_{N}=0, \\
{\left.[\cos \theta \operatorname{Re} \Omega+\sin \theta \operatorname{Im} \Omega]\right|_{N}=d \operatorname{Vol}_{N}}
\end{gathered}
$$

where the holomorphic 3-form is given by

$$
\Omega=Z^{\hat{K}} \alpha_{\hat{K}}-\mathcal{F}_{\hat{K}} \beta^{\hat{K}}, \quad J \wedge J \wedge J=\frac{3 i}{4} \Omega \wedge \bar{\Omega} .
$$

$\left(\alpha_{\hat{K}}, \beta^{\hat{K}}\right)$ is the dual cohomology basis of $\left(A^{\hat{K}}, B_{\hat{K}}\right)$ and the real basis of $H^{3}\left(\mathrm{CY}_{3}\right)$ in that they satisfy $\int_{\mathrm{CY}_{3}} \alpha_{\hat{K}} \wedge \beta^{\hat{L}}=$ $\delta_{\hat{K}}^{\hat{L}}$ with all other intersections vanishing.

We will consider D3-brane wrapping over a basis of the 3 cycle $A^{\hat{K}}$ or $B_{\hat{K}}\left(\hat{K}=0,1, \cdots, h^{2,1}\right)$ as the special Lagrangian submanifold. Taking into account (38), we assume the existence of special Lagrangian cycles defined by the following conditions:

$$
\begin{gathered}
\left.\operatorname{Re} \Omega\right|_{A^{\hat{K}}}=d \operatorname{Vol}_{A^{\hat{K}}},\left.\quad \operatorname{Im} \Omega\right|_{A^{\hat{K}}}=0, \quad\left(\theta_{A^{\hat{K}}}=0\right) \\
-\left.\operatorname{Im} \Omega\right|_{B_{\hat{K}}}=d \operatorname{Vol}_{B_{\hat{K}}},\left.\quad \operatorname{Re} \Omega\right|_{B_{\hat{K}}}=0, \quad\left(\theta_{B_{\hat{K}}}=-\frac{\pi}{2}\right) .
\end{gathered}
$$

Those conditions imply

$$
\Omega=\operatorname{Re} Z^{\hat{K}} \alpha_{\hat{K}}-i \operatorname{Im} \mathcal{F}_{\hat{L}} \beta^{\hat{L}}
$$

and

$$
\operatorname{Im} Z^{\hat{K}}=0, \quad \operatorname{Re} \mathcal{F}_{\hat{K}}=0 .
$$

Equations (43) and (E3) give rise to $\operatorname{Re} \mathcal{M}_{\hat{K} \hat{L}}=0$ and then the Hodge dual of $\left(\alpha_{\hat{K}}, \beta^{\hat{K}}\right)$ defined by (E1) and (E2) is

$$
* \alpha_{\hat{K}}=-(\operatorname{Im} \mathcal{M})_{\hat{K} \hat{L}} \beta^{\hat{L}}, \quad * \beta^{\hat{K}}=(\operatorname{Im} \mathcal{M})^{-1 \hat{K} \hat{L}} \alpha_{\hat{L}} .
$$

In general, a Hodge dual of a basis includes a linear combination of $\alpha_{\hat{K}}$ and $\beta^{\hat{K}}$ as (E1). On the other hand, Eq. (44) indicates that $\left(\alpha_{\hat{K}}, \beta^{\hat{K}}\right)$ is decomposed with respect to the Hodge dual, if D3-branes can wrap around $A^{\hat{K}}$ and $B_{\hat{K}}$ as special Lagrangian cycles with the conditions (40) and (41). By (42), (44), and (E4) $\left.* \operatorname{Re} \Omega\right|_{A^{\hat{K}}}=$ $\left.\operatorname{Im} \Omega\right|_{B_{\hat{K}}}$ is realized and then (40) and (41) give rise to $* d \operatorname{Vol}_{A^{\hat{K}}}=-d \operatorname{Vol}_{B_{\hat{K}}}$.

The world volume action of the wrapped D3-brane is described by

$$
\begin{aligned}
S_{\mathrm{D} 3} & =-T_{3} \int_{R \times A^{\hat{K}}} d^{3+1} \xi e^{-\phi} \sqrt{-\gamma} \\
& =-\int d t e^{n} \times\left(2 \pi \sqrt{\alpha^{\prime}}\right)^{3} T_{3} V_{\mathrm{CY}_{3}}^{-1 / 2} \operatorname{ReZ}^{\hat{K}}
\end{aligned}
$$

where

$$
\mathrm{Vol}_{A^{\hat{K}}}=\int_{A^{\hat{K}}} \operatorname{Re} \Omega=\int_{\mathrm{CY}_{3}} \operatorname{Re} \Omega \wedge \beta^{\hat{K}}=\operatorname{Re} Z^{\hat{K}} .
$$

Then the mass is given by

$$
m_{3}^{(\hat{K})}=\left(2 \pi \sqrt{\alpha^{\prime}}\right)^{3} T_{3} V_{\mathrm{CY}_{3}}^{-1 / 2} \operatorname{Re} Z^{\hat{K}} .
$$

The dual volume is also defined by

$$
\operatorname{Vol}_{B_{\hat{K}}}=\int_{B_{\hat{K}}}(-\operatorname{Im} \Omega)=-\int_{\mathrm{CY}_{3}} \operatorname{Im} \Omega \wedge \alpha_{\hat{K}}=-\operatorname{Im} \mathcal{F}_{\hat{K}}
$$

and the dual mass is

$$
m_{3,(\hat{K})}=-\left(2 \pi \sqrt{\alpha^{\prime}}\right)^{3} T_{3} V_{\mathrm{CY}_{3}}^{-1 / 2} \operatorname{Im} \mathcal{F}_{\hat{K}} .
$$

Using (42) and the following relation

$$
\begin{gathered}
4 V_{\mathrm{CY}_{3}}=\int_{\mathrm{CY}_{3}} \operatorname{Re} \Omega \wedge \operatorname{Im} \Omega=-\operatorname{Re} Z^{\hat{K}} \operatorname{Im} \mathcal{F}_{\hat{K}}, \\
m_{3}^{(\hat{K})} \text { and } m_{3,(\hat{K})} \text { satisfy } \\
\sum_{\hat{K} \in h^{2,1}+1} m_{3}^{(\hat{K})} \times m_{3,(\hat{K})}=4\left(\frac{1}{\sqrt{\alpha^{\prime}}}\right)^{2} .
\end{gathered}
$$

Those masses also have the following relation:

$$
m_{3,(\hat{K})}=-\operatorname{Im} \mathcal{M}_{\hat{K} \hat{L}} m_{3}^{(\hat{L})} .
$$

For $h^{1,2}+1>1$ the mass of the D3-brane can take various values. For example, we consider $Z^{1} \rightarrow 0$ and then massless D3-branes appear on the four-dimensional space-time [39]. If $\operatorname{Re} Z^{\hat{K}}$ has a scaling as $\exp (3 s) \operatorname{Re} Z^{\hat{K}}$ for a constant $s, \mathcal{F}_{\hat{K}}$ also has a scaling as $\exp (3 s) \mathcal{F}_{\hat{K}}$, because $\mathcal{F}$ is the holomorphic function with the degree two. Then, by (46)-(48) it is found that the mass is of order of the Planck mass for a large volume given by $\exp (3 s) \operatorname{Re} Z^{\hat{K}} \sim$ $\left(\mathcal{O}\left(10^{5}\right) \times 2 \pi \sqrt{\alpha^{\prime}}\right)^{3}$. This result corresponds to (24).

In Sec. IV and V, we have considered the scaling behavior of the mass of wrapped branes. For the string frame, a large volume of the compactified space and a weak string coupling give rise to heavy branes, while, in the fourdimensional Einstein frame, various light particles of the wrapped branes arise after the $T^{6}$ and the $\mathrm{CY}_{3}$ compactification. Those light particles are given by the string scale which is of order of the Planck length. For example, it has been shown that the mass of a D0-brane is of order $\mathcal{O}(10) \mathrm{TeV}$ for $V_{T^{6} \text { or } \mathrm{CY}_{3}} \sim\left(\mathcal{O}\left(10^{5}\right) \times 2 \pi \sqrt{\alpha^{\prime}}\right)^{6}$. Therefore, the four-dimensional Einstein frame is quite nontrivial. If the light particles have a weak RR interaction, the 
wrapped branes may become a component of the dark matter. We will discuss the RR charge in the next section.

\section{RR CHARGE IN THE FOUR-DIMENSIONAL EINSTEIN FRAME}

In Secs. IV and V, we have shown that various $\mathrm{D} p$-branes become light in the four-dimensional Einstein frame. The light $\mathrm{D} p$-brane locally interact through the $\mathrm{RR}$ flux. We have to estimate the RR charge, because the dark matter should have a very weak interaction. To estimate the coupling of the RR flux, we will analyze the RR flux and the Wess-Zumino (WZ) term by the four-dimensional Einstein frame. If the $\mathrm{D} p$-brane has a weak coupling, the light $\mathrm{D} p$-brane is a dark matter candidate. We do not consider a contribution of the D-instanton $(p=-1)$ potential, for simplicity. In this section, the RR potential is a function of the four-dimensional coordinates, while the fields on the $D p$-brane and the scale factor depend only on the time coordinate.

The action of the RR flux and the WZ term of a $D p$-brane wrapping over a $\left(m_{1} \cdots m_{p}\right)$ cycle is given by [31]

$$
\begin{aligned}
S_{\mathrm{RR}}^{\left(m_{1} \cdots m_{p}\right)} & =-\frac{1}{4 \kappa_{10}^{2}} \int_{M^{10}} d^{10} X \sqrt{-G}\left|F_{p+2}\right|^{2}, \\
S_{\mathrm{WZ}}^{\left(m_{1} \cdots m_{p}\right)} & =\mu_{p} \int_{R \times \Sigma_{p}} \exp \left(2 \pi \alpha^{\prime} F_{(2)}\right) \wedge \sum_{q} C_{q}
\end{aligned}
$$

where

$$
\left|F_{p+2}\right|^{2} \equiv \frac{1}{(p+2) !} F_{\mu_{1} \cdots \mu_{p+2}} F^{\mu_{1} \cdots \mu_{p+2}},
$$

$F_{p+2}=d C_{p+1}$ is the RR flux and $F_{(2)}$ is the gauge field on the D-brane. In the string frame the RR charge has a relation given by [31]

$$
\mu_{p}^{2}=T_{p}^{2}
$$

We will consider the dimensional reduction of the WZ term. The gauge field on the $\mathrm{D} p$-brane is given by

$$
F_{(2)}=\dot{A}_{a}(t) d t \wedge d \xi^{a} .
$$

This equation leads to a relation $F_{(2)} \wedge \cdots \wedge F_{(2)}=0$. The RR potential of a $\mathrm{D} p$-brane wrapping the specific $\left(m_{1} \cdots m_{p}\right)$ cycle can be expanded as

$$
\begin{aligned}
C_{p+1}= & C_{\mu}^{\left(m_{1} \cdots m_{p}\right)}(x) \frac{d X^{\mu}}{d t} d t \wedge d \xi^{m_{1}} \wedge \cdots \wedge d \xi^{m_{p}} \\
& +\sum_{b=p+1}^{6} C_{m_{b}}^{\left(m_{1} \cdots m_{p}\right)}(x) \frac{d X^{m_{b}}}{d t} d t \wedge d \xi^{m_{1}} \\
& \wedge \cdots \wedge d \xi^{m_{p}}
\end{aligned}
$$

where $X^{i}$ and $X^{m_{b}}$ are transverse coordinates of the wrapped $\mathrm{D} p$-brane. After the Kaluza-Klein reduction,
$C_{m_{b}}^{\left(m_{1} \cdots m_{p}\right)}(x)$ is a scalar field on the four-dimensional space-time and the WZ term is

$$
\begin{aligned}
S_{\mathrm{WZ}}^{\left(m_{1} \cdots m_{p}\right)}= & \int_{M_{4}} d^{4} x\left(2 \pi \sqrt{\alpha^{\prime}}\right)^{p} \mu_{p} \delta^{3}(\mathbf{x}-\mathbf{X}(t)) \\
& \times\left(C_{\mu}^{\left(m_{1} \cdots m_{p}\right)}(x) \frac{d X^{\mu}}{d t}\right. \\
& \left.+\sum_{b=p+1}^{6} C_{m_{b}}^{\left(m_{1} \cdots m_{p}\right)}(x) \frac{d X^{m_{b}}}{d t}\right) .
\end{aligned}
$$

First, we will consider the $T^{6}$ compactification. We perform the Kaluza-Klein reduction of the kinetic term of the RR flux and expand the RR potential on the tendimensional space-time as $C_{p+1}=\left(C_{\mu}^{\left(m_{1} \cdots m_{p}\right)} d x^{\mu}+\right.$ $\left.\sum_{b=p+1}^{6} C_{m_{b}}^{\left(m_{1} \cdots m_{p}\right)} d y^{m_{b}}\right) \wedge d y^{m_{1}} \wedge \cdots \wedge d y^{m_{p}}$. Substituting this equation for (51) and using (3), (5), (6), (8), and (54) we obtain the following effective action of the RR flux in the four-dimensional Einstein frame:

$$
\begin{aligned}
& S_{\mathrm{RR}}^{\left(m_{1} \cdots m_{p}\right)}= \int_{M^{4}} d^{4} x \sqrt{-g_{4}}\left(-\frac{1}{4\left(g_{p}^{\left(m_{1} \cdots m_{p}\right)}\right)^{2}} \tilde{F}_{\mu \nu}^{\left(m_{1} \cdots m_{p}\right)}\right. \\
& \times \tilde{F}^{\left(m_{1} \cdots m_{p}\right) \mu \nu}-\sum_{b=p+1}^{6} \frac{1}{2\left(\tilde{g}_{p}^{\left(m_{1} \cdots m_{p}, m_{b}\right)}\right)^{2}} \\
&\left.\times \partial_{\mu} \tilde{C}_{m_{b}}^{\left(m_{1} \cdots m_{p}\right)} \partial^{\mu} \tilde{C}_{m_{b}}^{\left(m_{1} \cdots m_{p}\right)}\right), \\
&\left(g_{p}^{\left(m_{1} \cdots m_{p}\right)}\right)^{2} \equiv 2 \pi \exp \left(-\bar{\lambda}+2 \sum_{a=1}^{p} \lambda_{m_{a}}\right), \\
&\left(\tilde{g}_{p}^{\left(m_{1} \cdots m_{p}, m_{b}\right)}\right)^{2} \equiv 2 \pi \exp \left(-2 \phi+2 \sum_{a=1}^{p} \lambda_{m_{a}}+2 \lambda_{m_{b}}\right)
\end{aligned}
$$

where

$$
\begin{aligned}
\tilde{F}_{\mu \nu}^{\left(m_{1} \cdots m_{p}\right)} & =\partial_{\mu} \tilde{C}_{\nu}^{\left(m_{1} \cdots m_{p}\right)}-\partial_{\nu} \tilde{C}_{\mu}^{\left(m_{1} \cdots m_{p}\right)}, \\
\tilde{C}_{\mu}^{\left(m_{1} \cdots m_{p}\right)} & \equiv\left(2 \pi \sqrt{\alpha^{\prime}}\right)^{p} \mu_{p} C_{\mu}^{\left(m_{1} \cdots m_{p}\right)}, \\
\tilde{C}_{m_{b}}^{\left(m_{1} \cdots m_{p}\right)} & \equiv\left(2 \pi \sqrt{\alpha^{\prime}}\right)^{p} \mu_{p} C_{m_{b}}^{\left(m_{1} \cdots m_{p}\right)} .
\end{aligned}
$$

The effective action of $\tilde{F}_{\mu \nu}^{\left(m_{1} \cdots m_{p}\right)}$ has no dependence of the dilaton. This is the fact on $N=2$ supergravity [40]. The dilaton field lives in a hypermultiplet and does not couple with a vector multiplet.

We find that $g_{p}^{\left(m_{1} \cdots m_{p}\right)}$ satisfies the electric-magnetic dual relation:

$$
g_{p}^{\left(m_{1} \cdots m_{p}\right)} \times g_{6-p}^{\left(m_{p+1} \cdots m_{6}\right)}=2 \pi .
$$

By (62) the coupling $g_{p}^{\left(m_{1} \cdots m_{p}\right)}$ can take a small value, while the dual coupling $g_{6-p}^{\left(m_{p+1} \cdots m_{6}\right)}$ becomes large. The dual 
relation (62) is also understood as follows. If we add a trivial quantity which is proportional to $\int F_{p+2} \wedge G_{8-p}$, $G_{8-p}=d D_{7-p}$ to the action (51) and eliminate $F_{p+2}$, we obtain $\int G_{8-p} \wedge * G_{8-p}$ by the dual field $G_{8-p}=* F_{p+2}$. Performing the Kaluza-Klein reduction, we can take the coupling of the dual field, $g_{6-p}^{2}$ in terms of the definition (59). We consider $\tilde{g}_{p}^{\left(m_{1} \cdots m_{p}, m_{b}\right)}$ later.

Second, we would like to discuss the $\mathrm{CY}_{3}$ compactification. The property considered above is general and it is expected that the charge relations can be satisfied in the cases of the $\mathrm{CY}_{3}$ compactification. However, in the $\mathrm{CY}_{3}$ compactification, the square of the coupling becomes a matrix defined by the Kähler or the complex structure moduli after the Kaluza-Klein reduction [40-44]. For example, we would like to consider the type IIA supergravity compactified on a $\mathrm{CY}_{3}$. After rescaling as

$$
C_{p+1}=\mu_{p}^{-1} \tilde{C}_{p+1}
$$

the RR flux is given by

$$
F_{2}=\mu_{0}^{-1} \tilde{F}, \quad F_{4}=\mu_{2}^{-1} \tilde{F}^{A} \omega_{A} .
$$

Then the gauge kinetic term of the vector multiplets is given by

$$
\begin{aligned}
-\frac{1}{2 \kappa_{10}^{2}} \int_{M_{10}} \frac{1}{2}\left(F_{2} \wedge * F_{2}+F_{4} \wedge * F_{4}\right) \\
=\int_{M_{4}}-\frac{1}{2}\left[\left\{\frac{-1}{2 \pi} \frac{\operatorname{Im} \mathcal{N}_{00}}{\left(2 \pi \sqrt{\alpha^{\prime}}\right)^{6}}\right\} \tilde{F} \wedge * \tilde{F}\right. \\
\left.+\left\{\frac{-1}{2 \pi} \frac{\operatorname{Im} \mathcal{N} \mathcal{N}_{A B}}{\left(2 \pi \sqrt{\alpha^{\prime}}\right)^{2}}\right\} \tilde{F}^{A} \wedge * \tilde{F}^{B}\right]
\end{aligned}
$$

where $\left(A=1, \cdots, h^{1,1}\right)$. $\operatorname{Im} \mathcal{N}_{00}$ and $\operatorname{Im} \mathcal{N}_{A B}$ are defined by (D5). Introducing a dual field $\tilde{G}_{6}=\tilde{F}_{A}^{\text {dual }} \tilde{\omega}^{A}$ and $\tilde{G}_{8}=$ $\tilde{F}^{\text {dual }} d \mathrm{Vol}_{\mathrm{CY}_{3}}$ which are related to the flux of the D4- and D6-branes, we will consider the following term:

$$
\begin{aligned}
& \frac{1}{2 \pi} \int_{M_{10}} \tilde{F}_{2} \wedge \tilde{G}_{8}=\frac{1}{2 \pi} \int_{M_{4}} V_{\mathrm{CY}_{3}} \tilde{F} \wedge \tilde{F}^{\text {dual }}, \\
& \frac{1}{2 \pi} \int_{M_{10}} \tilde{F}_{4} \wedge \tilde{G}_{6}=\frac{1}{2 \pi} \int_{M_{4}} \tilde{F}^{A} \wedge \tilde{F}_{A}^{\text {dual }}
\end{aligned}
$$

where we have used (D7). Adding (64) to (63) and integrating out $\tilde{F}$ and $\tilde{F}^{A}\left(A=1,2, \cdots, h^{1,1}\right)$, the gauge kinetic term of (63) is mapped to

$$
\begin{aligned}
\int_{M_{4}}-\frac{1}{2} & {\left[\left\{\frac{-1}{2 \pi}\left(2 \pi \sqrt{\alpha^{\prime}}\right)^{6}\left(\operatorname{Im} \mathcal{N}^{-1}\right)^{00}\right\} \tilde{F}^{\text {dual }} \wedge * \tilde{F}^{\text {dual }}\right.} \\
+ & \left.\left\{\frac{-1}{2 \pi}\left(2 \pi \sqrt{\alpha^{\prime}}\right)^{2}\left(\operatorname{Im} \mathcal{N}^{-1}\right)^{A B}\right\} \tilde{F}_{A}^{\text {dual }} \wedge * \tilde{F}_{B}^{\text {dual }}\right]
\end{aligned}
$$

where the inverse matrix on the couplings is defined by (D6) and $*^{2} \tilde{F}_{\hat{A}}^{\text {dual }}=-\tilde{F}_{\hat{A}}^{\text {dual }}$ has been used for a fourdimensional Lorentz manifold. We can read off the gauge couplings is as follows:

$$
\begin{aligned}
& \left(g^{-2}\right)_{00}=\frac{-1}{2 \pi} \frac{\operatorname{Im} \mathcal{N}_{00}}{\left(2 \pi \sqrt{\alpha^{\prime}}\right)^{6}}, \\
& \left(g^{-2}\right)_{A B}=\frac{-1}{2 \pi} \frac{\operatorname{Im} \mathcal{N}_{A B}}{\left(2 \pi \sqrt{\alpha^{\prime}}\right)^{2}}, \\
& \left(g_{\text {dual }}^{-2}\right)^{00}=\frac{-1}{2 \pi}\left(2 \pi \sqrt{\alpha^{\prime}}\right)^{6}\left(\operatorname{Im} \mathcal{N}^{-1}\right)^{00}, \\
& \left(g_{\text {dual }}^{-2}\right)^{A B}=\frac{-1}{2 \pi}\left(2 \pi \sqrt{\alpha^{\prime}}\right)^{2}\left(\operatorname{Im} \mathcal{N}^{-1}\right)^{A B} .
\end{aligned}
$$

Those couplings satisfy

$$
\left(g^{-2}\right)_{\hat{A} \hat{B}}\left(g_{\text {dual }}^{-2}\right)^{\hat{B} \hat{C}}=(2 \pi)^{-2} \delta_{\hat{A}}^{\hat{C}}
$$

where $\left(\hat{A}=0,1,2, \cdots, h^{1,1}\right)$. The above relation has the same structure to (62). In type IIB theory, the coupling of the flux of a D3-brane wrapping over a 3 cycle is given by replacing $\mathcal{N}$ with $\mathcal{M}$ defined by (E3) [44] and taking a suitable normalization constant.

We will consider the scaling behavior of $g_{p}^{\left(m_{1} \cdots m_{p}\right)}$ and $\tilde{g}_{p}^{\left(m_{1} \cdots m_{p}, m_{b}\right)}$, assuming a constant dilaton and constant moduli fields. We should notice that $\tilde{g}_{p}^{\left(m_{1} \cdots m_{p}, m_{b}\right)}$ is not the true coupling of $C_{m_{b}}^{\left(m_{1} \cdots m_{p}\right)}$, since the kinetic term of $X^{m_{b}}(t)$ has a coupling with moduli fields such as $e^{-2 \beta+2 \lambda^{\prime}}\left(d X^{m_{b}} / d t\right)^{2}$ in (10). To normalized the kinetic term, we define the following equations:

$$
\frac{d X^{m_{b}}}{d t} \equiv e^{\beta-\lambda_{m_{b}}} \frac{d \tilde{X}^{m_{b}}}{d t}, \quad \tilde{C}_{m_{b}}^{\left(m_{1} \cdots m_{p}\right)} \equiv e^{-\beta+\lambda_{m_{b}}} \Psi_{m_{b}}^{\left(m_{1} \cdots m_{p}\right)}
$$

Then, the kinetic term is proportional to $e^{-2 n}\left(d \tilde{X}^{m_{b}} / d t\right)^{2}$ in (9). Using the above relations, the kinetic term of $\Psi_{m_{b}}^{\left(m_{1} \cdots m_{p}\right)}$ is given by

$$
\sum_{b=p+1}^{6} \frac{-1}{2\left(g_{p}^{\left(m_{1} \cdots m_{p}, m_{b}\right)}\right)^{2}} \partial_{\mu} \Psi_{m_{b}}^{\left(m_{1} \cdots m_{p}\right)} \partial^{\mu} \Psi_{m_{b}}^{\left(m_{1} \cdots m_{p}\right)}
$$

where the coupling corresponds with (59):

$$
\left(g_{p}^{\left(m_{1} \cdots m_{p}\right)}\right)^{2}=\left(g_{p}^{\left(m_{1} \cdots m_{p}, m_{b}\right)}\right)^{2} .
$$

We will consider the isotropic case such as $\lambda_{m}=\lambda_{n}$. $g_{p}^{\left(m_{1} \cdots m_{p}\right)}$ and $g_{p}^{\left(m_{1} \cdots m_{p}, m_{b}\right)}$ can take a small value for $p \leq 2$ and $e^{\lambda^{\prime}} \gg 1$. We will take $e^{\lambda^{\prime}} \sim \mathcal{O}\left(10^{5}\right)$ for instance. The coupling is given by

$$
\left(g_{p}^{\left(m_{1} \cdots m_{p}\right)}\right)^{2}=\left(g_{p}^{\left(m_{1} \cdots m_{p}, m_{b}\right)}\right)^{2} \sim \mathcal{O}\left(10^{10(p-3)}\right) .
$$

According to the relation, D0-brane has the very weak interaction $g_{(0)}^{2} \sim \mathcal{O}\left(10^{-30}\right)$. For wrapped D1- and D2branes the coupling is given by $\left(g_{1}^{\left(m_{1}\right)}\right)^{2} \sim \mathcal{O}\left(10^{-20}\right)$ and $\left(g_{2}^{\left(m_{1} m_{2}\right)}\right)^{2} \sim \mathcal{O}\left(10^{-10}\right)$, respectively. The coupling of D3brane has $g_{3}^{\left(m_{1} m_{2} m_{3}\right)} \sim \mathcal{O}(1)$. In the anisotropic case, $\lambda_{m} \neq$ $\lambda_{n}$, Eq. (59) with (62) has the same classification on the mass of D-branes in Sec. IV. 
We also investigate scaling of the charges, controlling scales of the Calabi-Yau by hand. In the case with a $\mathrm{CY}_{3}$ compactification small couplings of wrapped D0- and D2branes are realized by a large $-\operatorname{Im} \mathcal{N}_{\hat{A} \hat{B}}$, because of $-\operatorname{Im} \mathcal{N}_{\hat{A} \hat{B}} \propto\left(g^{-2}\right)_{\hat{A} \hat{B}}$. For example, using (4) and (5), we consider a large volume of the $\mathrm{CY}_{3}$ by a scaling, $v^{A} \rightarrow$ $\exp (2 s) v^{A}$ for a positive constant $s$. This condition gives rise to

$$
\begin{aligned}
\operatorname{Im} \mathcal{N}_{00} & \rightarrow \exp (6 s) \operatorname{Im} \mathcal{N}_{00} \\
\operatorname{Im} \mathcal{N}_{A B} & \rightarrow \exp (2 s) \operatorname{Im} \mathcal{N}_{A B}
\end{aligned}
$$

Using (66) and (D5), the coupling of the D0-brane can be $g^{2} \sim \mathcal{O}\left(10^{-30}\right)$ for $V_{\mathrm{CY}_{3}} \sim\left(\mathcal{O}\left(10^{5}\right) \times 2 \pi \sqrt{\alpha^{\prime}}\right)^{6}$. Then the weak coupling is realized for wrapped D0- and D2-branes. The coupling of D3-branes which is related with the matrix $\mathcal{M}_{\hat{K} \hat{L}}$ in (E3) has different behavior from the flux of type IIA. The coupling involves the holomorphic function with the degree two, $\mathcal{F}\left(\exp \left(3 s^{\prime}\right) Z^{\hat{K}}\right)=\exp \left(6 s^{\prime}\right) \mathcal{F}\left(Z^{\hat{K}}\right)$ by $Z^{\hat{K}} \rightarrow \exp \left(3 s^{\prime}\right) Z^{\hat{K}}$. Then, (E3) implies that the coupling matrix $\mathcal{M}$ does not change for the scaling of $Z^{\hat{K}}$. We may require details of the topological data of the $\mathrm{CY}_{3}$ to analyze the scaling of $\mathcal{M}$, however we do not consider the details in this paper.

\section{FLUCTUATIONS OF MODULI FIELDS}

In previous sections, we have investigated the mass and the RR charge of the wrapped branes to consider a possibility of a dark matter candidate. It has been shown that there are cases where the wrapped $\mathrm{D} p$-brane has a light mass and a weak RR charge in the four-dimensional Einstein frame, if the scale of the compactified space satisfies a condition discussed in Secs. IV, V, and VI. However, we have to discuss an interaction in terms of fluctuations of moduli fields, because the fluctuations give rise to propagations of scalar interactions between branes. In fact, from the ten-dimensional point of view, the radial moduli is one of components of the gravitational fields and then the fluctuations of the moduli also give the interaction between the D-branes [31]. In this section, we would like to comment on a charge of the interaction without the analysis of the cosmological perturbation. We consider the $T^{6}$ compactification only.

The kinetic term of the moduli fields is diagonal in (4). To canonically normalize the kinetic term of the fluctuations, we define

$$
\lambda_{m}=\lambda_{m}^{(0)}+\sqrt{8 \pi G_{4}} \delta \tilde{\lambda}_{m}
$$

where $\lambda_{m}^{(0)}$ is a fixed constant. Then, using (9), the source term of the interaction is given by

$$
\begin{aligned}
\delta S_{\mathrm{D} p}^{\left(m_{1} \cdots m_{p}\right)} \propto & -\left(2 \pi \sqrt{\alpha^{\prime}}\right)^{p} T_{p} \sqrt{8 \pi G_{4}} \exp \left(\sum_{a=1}^{p} \lambda_{m_{a}}^{(0)}-\frac{\bar{\lambda}^{(0)}}{2}\right) \\
& \times \int d t e^{n(t)}\left(-\frac{1}{2} \delta \overline{\tilde{\lambda}}+\sum_{a=1}^{p} \delta \tilde{\lambda}_{m_{a}}\right) .
\end{aligned}
$$

The coefficient of the above equation gives the charge in terms of the fluctuation. The magnitude of the interaction is given by the square of the coefficient:

$$
\pi \exp \left(2 \sum_{a=1}^{p} \lambda_{m_{a}}^{(0)}-\bar{\lambda}^{(0)}\right)
$$

It is found that the scaling of (75) is the same to (59) and the similar dual relation such as (62) is satisfied. If $2 \sum_{a=1}^{p} \lambda_{m_{a}}^{(0)}-\bar{\lambda}^{(0)}<0$ is satisfied, the charge becomes weak. A condition, $\lambda_{m}^{(0)}=\lambda_{n}^{(0)}$ also realizes a weak interaction on the fluctuations at a large scale of $T^{6}$ for D0-, D1-, and D2-branes.

Taking into account the results discussed in previous sections, we find that there are cases in which wrapped $\mathrm{D} p$-branes have the light mass, the weak RR charge, and the weak interaction on the fluctuations of the moduli fields in the four-dimensional Einstein frame, considering a large volume of the compactified space. For instance, the mass of a D0-brane is of the order of $\mathcal{O}(10) \mathrm{TeV}$ for $V_{T^{6}{ }_{\text {or }} \mathrm{CY}_{3}} \sim$ $\left(\mathcal{O}\left(10^{5}\right) \times 2 \pi \sqrt{\alpha^{\prime}}\right)^{6}$. The square of the charge on the RR flux and the fluctuation of the moduli fields is also of order $\mathcal{O}\left(10^{-30}\right)$. Therefore, a possibility of the dark matter arises from the wrapped $\mathrm{D} p$-branes in the four-dimensional Einstein frame.

\section{D1-KK5 BRANE GAS SYSTEM}

We have considered the behavior of masses and charges of wrapped branes in the four-dimensional Einstein frame, adjusting the compactification scale by hand. However, it is not necessarily possible to tune the scale of the compactification freely, because this tuning should be consistent with a weak string coupling to consider the effective field theory at a low energy after the moduli stabilization. In this section, we will investigate a moduli stabilization in the $T^{6}$ compactification, using the brane gas system of D1-branes and KK5-monopoles in type IIB string theory [25]. A condition for a large volume and a weak string coupling will be explained. We will estimate the mass of the wrapped branes.

At a matter-dominated era, the velocity of wrapped branes may vanish, on average, in a very large scale. Then, we will consider the case of $\dot{X}^{i}(t) \simeq 0$ which means that wrapped brane gases are completely nonrelativistic particles [25]. We have assumed the cancellation of the total charge under the brane gas approximation and then we can use the results in Sec. III. We analyze a type IIB brane gas model in which the D1-branes wrap over each 1 cycle and the KK5-monopoles wrap over the (45678) 
cycle and its cyclic permutations. We will take the following initial condition,

$$
f_{m_{a}}=2 \pi \alpha^{\prime} f, \quad v^{m_{a}}=0
$$

to obtain the analytic value of $\lambda_{a}(t)$ and $\beta(t)$ at a minimum. The choice of this initial condition represents the fact where the initial gauge fields on the D1-branes are the same for each cycle. This condition may be natural for the isotropic expansion of the internal space. The above condition provides us the following energy density:

$$
U^{\mathrm{IIB}}=e^{-3 A(t)} \times\left(N_{\mathrm{D} 1} m_{1}+N_{\mathrm{KK} 5} m_{\mathrm{KK} 5}\right)
$$

where $N_{\mathrm{D} 1}$ and $N_{\mathrm{KK} 5}$ are the number density of the point particles. This number density is a constant because the number density is decided at present as $e^{A(t=0)}=1$. The scaling of the number density is controlled by the scale factor $e^{A(t)} \cdot m_{\mathrm{D} 1}$ and $m_{\mathrm{KK} 5}$ are given by

$$
m_{1} \equiv\left(2 \pi \sqrt{\alpha^{\prime}}\right) T_{1} \sum_{a=4}^{9} e^{-(1 / 2) \bar{\lambda}(t)+\lambda_{a}(t)}\left(1+f^{2} e^{2 \beta(t)+\bar{\lambda}}\right)^{1 / 2},
$$

$$
m_{\mathrm{KK} 5} \equiv\left(2 \pi \sqrt{\alpha^{\prime}}\right)^{5} T_{\mathrm{KK} 5} \sum_{a=4}^{9} e^{-\beta(t)+\lambda_{a}(t)} .
$$

If all moduli fields are fixed, $U^{\mathrm{IIB}}$ is equal to the energy density of a pressureless matter as $\rho \sim e^{-3 A}$.

By (78) and (79) $U^{\mathrm{IIB}}$ takes a positive value. Thus the minimum is given by $\partial U^{\mathrm{IIB}} / \partial \beta=\partial U^{\mathrm{IIB}} / \partial \lambda_{a}=0$. $\left(\frac{\partial}{\partial \lambda_{a}}-\right.$ $\left.\frac{\partial}{\partial \lambda_{b}}\right) U^{\mathrm{IIB}}=0$ gives

$$
e^{\lambda_{a}(t)}=e^{\lambda_{b}(t)} \equiv e^{\lambda^{\prime}(t)}
$$

for $(a, b=4,5, \ldots, 9)$. Using (80) and the minimum conditions, we obtain analytic values of moduli fields at the minimum [25]:

$$
\begin{gathered}
e^{2 \beta_{\text {min. }}}=\sqrt{\frac{2}{f^{2}}} \frac{N_{\mathrm{KK} 5}}{N_{\mathrm{D} 1}}, \\
e^{2 \lambda_{\text {min. }}^{\prime}}=\left(\frac{1}{2 f^{2}}\right)^{1 / 6}\left(\frac{N_{\mathrm{D} 1}}{N_{\mathrm{KK} 5}}\right)^{1 / 3} .
\end{gathered}
$$

As shown in Sec. III, $f$ is an integer, therefore the stabilized value of moduli fields cannot continuously connect to another stabilized value under the $T^{6}$ compactification.

These equations have a relation as $e^{2 \phi_{\min }}=$ $e^{2 \beta_{\min } .}+6 \lambda_{\min .}^{\prime}=f^{-2}$. The dilaton field at the minimum is given by

$$
e^{2 \phi_{\min }}=\frac{1}{f^{2}}
$$

where we have used (3), (81), and (82). By (82) and (83) there is a relation between $N_{\mathrm{D} 1}$ and $N_{\mathrm{KK} 5}$ as follows:

$$
\frac{N_{\mathrm{D} 1}}{N_{\mathrm{KK} 5}}=\sqrt{2} e^{-\phi_{\min .}+6 \lambda_{\min .}^{\prime}} .
$$

If a large volume of the $T^{6}, \exp \left(\lambda_{\text {mim }}^{\prime}\right) \gg 1$ and a weak string coupling,

$$
g_{s}=\exp \left(\phi_{\text {mim. }}\right)=\sqrt{\frac{1}{f^{2}}} \ll 1
$$

are imposed at the minimum, (84) gives the following condition:

$$
N_{\mathrm{D} 1} \gg N_{\mathrm{KK} 5} .
$$

Equation (86) indicates that the wrapped D1-brane gas dominates the components. The number density of KK5 has very low number density by the relation (84) and then KK5-monopoles may survive the annihilation by the heavy mass and the low number density on the three-dimensional space.

Equations (82) and (83) show that the string coupling $g_{s}=e^{\phi_{\text {min. }}}$. is related to the scale of the compactification through the initial condition of the electric fields on the D1brane. We require a weak string coupling $g_{s} \ll 1$ and a large scale of the compactification $e^{\lambda_{\min }^{\prime}} \gg 1$. If we take the specific initial condition as $1 \ll f^{2}$ in (83), the weak string coupling is realized. However, Dirac-Born-Infeld action has upper bound for the electric fields. This bound is given by

$$
1-\mathcal{A}(t)>0
$$

in (9) because of $\dot{X}^{i}(t) \simeq 0$. We have to check that the initial condition satisfies (87) at the minimum. Substituting (81) and (82) for (13), we obtain the following condition for a D1-brane:

$$
1-\mathcal{A}_{\text {min. }}=1-\frac{f^{2} e^{2 \beta_{\text {min. }}+6 \lambda_{\min .}^{\prime}}}{1+f^{2} e^{2 \beta_{\min .}}+6 \lambda_{\min .}^{\prime}}=\frac{1}{2} .
$$

Therefore we can take the initial condition satisfying $1 \ll$ $f^{2}$ because (88) does not depend on any initial condition in this model. By (84) and (87) we can take the weak string coupling and the large volume of $T^{6}$ simultaneously.

Finally, we would like to estimate the mass scale of the branes. At the minimum given by (82) and (83), the scale of $T^{6}$ is isotropic, i.e., $e^{\lambda_{a \min }}=e^{\lambda_{\min }^{\prime}}$ for $(a=4, \cdots, 9)$. Then, the mass of one of the D1-branes and KK5monopoles defined by (78) and (79) is given by

$$
\begin{aligned}
\left.m_{1}^{\left(m_{1}\right)}\right|_{\text {min. }} & =\frac{e^{-2 \lambda_{\text {min. }}^{\prime}}}{2} \times m_{\text {Planck }}, \\
\left.m_{\mathrm{KK} 5}^{\left(m_{1} \cdots m_{5}\right)}\right|_{\text {min. }} & =\frac{e^{-\phi_{\text {min. }}+4 \lambda_{\text {min. }}^{\prime}}}{2 \sqrt{2}} \times m_{\text {Planck }}
\end{aligned}
$$

where $m_{\text {Planck }} \equiv G_{4}^{-1 / 2}=2 \sqrt{2} / \sqrt{\alpha^{\prime}} \sim \mathcal{O}\left(10^{19}\right) \mathrm{GeV}$ is the four-dimensional Planck mass. If we take a weak string coupling $e^{\phi_{\text {min. }}} \ll 1$ and the large scale, $e^{\lambda_{\text {min. }}^{\prime}} \gg 1$, the mass of the KK5-monopole becomes heavier than the 
Planck mass. On the other hand, D1-branes become light particles under the weak string coupling and the large scale of $T^{6}$. If we consider $e^{\lambda_{\text {min. }}^{\prime}} \sim \mathcal{O}\left(10^{5}\right)$, the order of the mass is given by $\left.m_{1}\right|_{\text {min. }} \sim \mathcal{O}\left(10^{9}\right) \mathrm{GeV}$.

In the present section, we have considered the brane gas model constructed by D1-KK5 as a simple model of the moduli stabilization. It is found that (85) and (86) are required to realize a large volume and a weak string coupling at a minimum of the potential and the D1-brane gas becomes light.

\section{SUMMARY AND DISCUSSIONS}

We have considered a possibility of the dark matter candidate for wrapped branes in brane gas cosmologies based on the type II string theories. Using the fourdimensional Einstein frame, we have investigated the mass, the RR charge, and the interaction on fluctuations of the moduli fields. This analysis has been done by the description of the effective field theory, taking the string scale as $1 / \sqrt{\alpha^{\prime}} \sim m_{\text {Planck. }}$. A large volume of the compactified space and a weak string coupling are required to suppress string excitations and to obtain the perturbative description.

We have found models where D-branes wrapping over cycles of a compactified space has a light mass by the large volume and the weak string coupling, after the $T^{6}$ and the $\mathrm{CY}_{3}$ compactification, while the mass becomes very heavy in the string frame. The masses satisfy the electricmagnetic dual relation between a $\mathrm{D} p$ - and a $\mathrm{D}(6-$ $p$ )-brane. The four-dimensional Einstein frame gives rise to the dual relation which is time independent although each mass depends on the time coordinate through moduli fields. The string and the $(d+1)$-dimensional $(d \neq 3)$ Einstein frame, on the other hand, derive the dual relation depending on the time variable. Thus, the four-dimensional Einstein frame is the special case and the electric-magnetic dual relation gives the mass hierarchy. For example, the mass of a D0-brane is of order $\mathcal{O}(10) \mathrm{TeV}$, if we take a large volume as $V_{T^{6} \text { or } \mathrm{CY}_{3}} \sim\left(\mathcal{O}\left(10^{5}\right) \times 2 \pi \sqrt{\alpha^{\prime}}\right)^{6}$. Similar dual relation, which is time independent, is realized for (F1, NS5) and for (the momentum of F1, KK5) for the $T^{6}$ compactification. The effective charges of the RR flux and of the moduli fluctuation have been investigated. The charges between the $\mathrm{D} p$ - and $\mathrm{D}(6-p)$-brane also satisfy the electric-magnetic dual relations and then there are cases in which the wrapped $\mathrm{D} p$-branes obtain a small charge for the large volume and the weak string coupling. For instance, the square of the charge of a D0-brane is of order $\mathcal{O}\left(10^{-30}\right)$ for $V_{T^{6} \text { or } \mathrm{CY}_{3}} \sim\left(\mathcal{O}\left(10^{5}\right) \times 2 \pi \sqrt{\alpha^{\prime}}\right)^{6}$.

We have considered the behavior of the mass of wrapped branes, using a toy model constructed by a D1-KK5 brane gas [25] where radial moduli fields of the $T^{6}$ and the dilaton are simultaneously stabilized. The effective field description requires a large volume of the compactified space and a weak string coupling, however a point where all moduli fields are stabilized depends on models and the realization of the condition is not trivial. In this model, the condition of the large volume and the weak string coupling imposes (85) and (86) at a minimum of the potential of the moduli fields and then light D1-branes appear for a large volume of the $T^{6}$.

Taking the four-dimensional Einstein frame is very simple, however quite nontrivial results among masses and the charges are realized. We should consider the light wrapped branes in string cosmologies because of the very small charges. Those ingredients may be a candidate for the dark matter. We have considered no -Schwarz NevueSchwarz 2-form and no quantum correction. Those quantities may give the wrapped branes interesting results, using explicit $\mathrm{CY}_{3}$ compactifications.

To check the possibility on the dark matter more rigorously we have to investigate the density perturbation of the dust of wrapped branes. If domains with dense gases appear on the three-dimensional space, interactions on the RR flux and on the moduli fluctuations may have an interesting role for the evolution of the density, because of $\mathcal{O}\left(\left(g_{p}^{\left(m_{1} \cdots m_{p}\right)}\right)^{2}\right) \sim \mathcal{O}\left(G_{4}\left(m_{p \text {,winding }}^{\left(m_{1} \cdots m_{p}\right)}\right)^{2}\right)$ by (5), (16), and (59). The density perturbation has been considered in various brane gas models [12,27-29,45,46].

In the brane world model, the wrapped branes distribute in a bulk space as well as on the brane expanding the three dimensions. Then, various bound states may arise between many branes and the dark matter of wrapped branes may have interactions with the dark energy through the couplings of moduli fields. It may be interesting to investigate the dynamics of the Universe with the light branes in the four-dimensional Einstein frame for various models [4765].

\section{ACKNOWLEDGMENTS}

The authors thank the Yukawa Institute for Theoretical Physics at Kyoto University. Discussions during the YITP workshop YITP-W-08-04 on "Development of Quantum Field Theory and String Theory" were useful to complete this work. We also thank Particle Theory Group of the Yukawa Institute for Theoretical Physics for fruitful discussions. We would like to thank Tohru Eguchi, Kenji Hotta, Tetsuji Kimura, Hideo Kodama, Shinji Mukohyama, Misao Sasaki, Naoki Sasakura, Seiji Terashima for useful and helpful comments. M. S. is supported by a Sasagawa Scientific Research Grant from The Japan Science Society and by the Hokkaido University Clark Memorial Foundation.

\section{APPENDIX A: $(d+1)$-DIMENSIONAL EINSTEIN FRAME}

We will decompose the ten-dimensional space-time as $10=(d+1)+(10-(d+1))$ and take the $(d+1)-$ 
dimensional Einstein frame. The $(d+1)$-dimensional Einstein frame is obtained by a toroidal compactification of the circumference of $2 \pi \sqrt{\alpha^{\prime}} e^{\lambda_{m}}$ as

$$
\begin{aligned}
& \frac{1}{16 \pi G_{10}} \int d^{10} X \sqrt{-G} e^{-2 \phi} R \\
& =\frac{1}{16 \pi G_{d+1}} \int d^{d+1} x \sqrt{-g_{d+1}} R_{d+1}+\cdots
\end{aligned}
$$

where the $(d+1)$-dimensional gravitational constant is given by $G_{d+1}=G_{10} /\left(2 \pi \sqrt{\alpha^{\prime}}\right)^{10-(d+1)}$. To obtain this effective action we will consider the following variables:

$$
\begin{gathered}
G_{\mu \nu}=e^{2 \beta_{d+1}} g_{d+1, \mu \nu}, \quad G_{m n}=e^{2 \lambda_{m}} \delta_{m n}, \\
\beta_{d+1}=\frac{2}{d-1} \phi-\frac{1}{d-1} \sum_{m=d+1}^{9} \lambda_{m},
\end{gathered}
$$

where $\quad(\mu, \nu=0,1, \cdots, d) \quad$ and $\quad(m, n=d+1, d+$ $2, \cdots, 9)$. Equation (A2) defines the $(d+1)$-dimensional Einstein frame. If we consider $d=3$ and $\beta_{3+1} \equiv \beta$, the four-dimensional Einstein frame defined by (3) is recovered.

\section{APPENDIX B: $S$ DUALITY IN THE FOUR-DIMENSIONAL EINSTEIN FRAME}

The $S$-duality rule is defined by the ten-dimensional Einstein frame. Using (A2) in the case of $d=9$, we obtain

$$
G_{A B}=e^{2 \beta_{10}} g_{10, A B}, \quad \beta_{10}=\frac{1}{4} \phi=\frac{1}{4}\left(\beta+\frac{\bar{\lambda}}{2}\right)
$$

where $(A, B=0,1, \cdots, 9) . \beta_{10}$ is related with variables $(\beta, \bar{\lambda})$ which are defined by (3) as (B1). Then the $S$ duality is given by $\phi \rightarrow-\phi$ which means that the string-frame metric transforms as

$$
G_{A B} \rightarrow e^{-\phi} G_{A B} .
$$

To find the transformation rule of the $S$ duality in the fourdimensional Einstein frame we will assume

$$
\lambda_{m} \rightarrow \lambda_{m}+f_{\text {S-dual }}, \quad \beta \rightarrow \beta+f_{\text {S-dual }} .
$$

If this transformation represents the $S$ duality satisfying (B2), the action (4) must be invariant under this transformation. Substituting (B3) for (4), the $S$ dual invariance requires

$$
f_{\text {S-dual }}=-\frac{1}{2} \phi .
$$

It is found that this solution satisfies (B2), substituting (B4) for (B1).

\section{APPENDIX C: FUNDAMENTAL STRING GAS}

If the fundamental string wraps over a cycle of $T^{6}$ and we choose $\sigma^{0}=t, 0 \leq \sigma^{1} \leq 2 \pi \sqrt{\alpha^{\prime}}$, we can assume

$$
\begin{gathered}
X^{0}=t, \quad X^{m_{a}}=X^{m_{a}}(t)+w^{m_{a}} \sigma^{1}, \\
(a=1,2,3, \cdots, 6),
\end{gathered}
$$

where we have considered the only zero mode and $w^{m_{a}}$ indicates the winding number of the string. The above assumption is motivated by $X^{m_{a}}\left(t, \sigma^{1}+2 \pi \sqrt{\alpha^{\prime}}\right)=$ $X^{m_{a}}\left(t, \sigma^{1}\right)+2 \pi \sqrt{\alpha^{\prime}} w^{m_{a}}$. We do not consider a dependence of $X^{i}(i=1,2,3)$, for simplicity. The induced metric is given by

$$
\begin{aligned}
& \gamma_{00}=-e^{2 \lambda_{0}}+\sum_{a=1}^{6} e^{2 \lambda_{m_{a}}}\left(\dot{X}^{m_{a}}\right)^{2},
\end{aligned}
$$

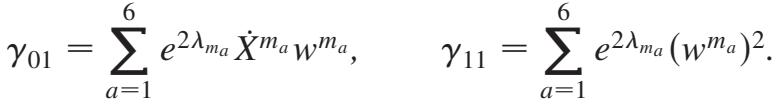

We will impose $\gamma_{0 m_{1}}=0$, the meaning of which is explained later. Then the world volume action of a fundamental string wrapping over a cycle of $T^{6}$ is given by

$$
\begin{aligned}
S_{\mathrm{F} 1}= & -T_{\mathrm{F} 1} \int_{R \times \Sigma_{1}} d \sigma^{0} d \sigma^{1} \sqrt{-\gamma} \\
= & -\left(2 \pi \sqrt{\alpha^{\prime}}\right) T_{\mathrm{F} 1} \int d t e^{\lambda_{0}}\left\{\sum_{a=1}^{6} e^{2 \lambda_{m_{a}}\left(w^{m_{a}}\right)^{2}}\right. \\
& \left.\times\left(1-\sum_{a=1}^{6} e^{2 \lambda_{m_{a}}-2 \lambda_{0}}\left(\dot{X}^{m_{a}}\right)^{2}\right)\right\}^{1 / 2} \\
= & -\left(2 \pi \sqrt{\alpha^{\prime}}\right) T_{\mathrm{F} 1} \int d t e^{\beta+n}\left\{\sum_{a=1}^{6} e^{2 \lambda_{m_{a}}\left(w^{m_{a}}\right)^{2}}\right. \\
& \left.\times\left(1-\sum_{a=1}^{6} e^{2 \lambda_{m_{a}}-2 \beta-2 n}\left(\dot{X}^{m_{a}}\right)^{2}\right)\right\}^{1 / 2}
\end{aligned}
$$

where $T_{\mathrm{F} 1}=T_{\mathrm{D} 1}$. The equation of motion is

$$
\begin{gathered}
\left(\sum_{a=1}^{6} e^{2 \lambda_{m_{a}}}\left(w^{m_{a}}\right)^{2}\right)^{1 / 2} e^{n+\beta} e^{2 \lambda_{m_{a}}-2 \beta-2 n} \dot{X}^{m_{a}} \\
\quad=n_{m_{a}}\left\{1-\sum_{a=1}^{6} e^{2 \lambda_{m_{a}}-2 \beta-2 n}\left(\dot{X}^{m_{a}}\right)^{2}\right\}^{1 / 2}
\end{gathered}
$$

where $n_{m_{a}}$ is a constant of integration. By the definition of the energy-momentum tensor (14) and (C4), we obtain

$$
\begin{aligned}
u_{\mathrm{F} 1}= & e^{-3 A} m_{\mathrm{F} 1} \\
= & e^{-3 A}\left(2 \pi \sqrt{\alpha^{\prime}}\right) T_{\mathrm{F} 1}\left\{\sum_{a=1}^{6} e^{2 \beta+2 \lambda_{m_{a}}\left(w^{m_{a}}\right)^{2}}\right. \\
& \left.+\sum_{a=1}^{6} e^{2 \beta-2 \lambda_{m_{a}}}\left(n_{m_{a}}\right)^{2}\right\}^{1 / 2} .
\end{aligned}
$$

Substituting (C4) for $\gamma_{0 m_{1}}=0$, we obtain 


$$
\sum_{a=1}^{6} n_{m_{a}} w^{m_{a}}=0
$$

If $n_{m_{a}}$ and $w^{m_{a}}$ are integers, (C6) indicates the level matching condition, $L_{0}-\tilde{L}_{0}=0$, for the only zero mode. The mass $m_{\mathrm{F} 1}$ is invariant under the $T$ duality, $\left(\lambda_{m_{a}} \rightarrow-\lambda_{m_{a}}\right.$, $\left.\phi \rightarrow \phi-\lambda_{m_{a}}\right)$ with $\left(n_{m_{a}} \leftrightarrow w^{m_{a}}\right)$.

\section{APPENDIX D: KÄHLER FORM}

The Kähler form $J$ is expanded by a harmonic form $\omega_{A}$ which is a basis of $H^{1,1}\left(\mathrm{CY}_{3}\right)$ as

$$
J=v^{A} \omega_{A}
$$

where $\left(A=1, \cdots, h^{1,1}\right)$. It is useful to define the following quantities:

$$
\begin{aligned}
\mathcal{K}_{A B C} & =\int_{\mathrm{CY}_{3}} \omega_{A} \wedge \omega_{B} \wedge \omega_{C}, \\
\mathcal{K}_{A B} & =\int_{\mathrm{CY}_{3}} \omega_{A} \wedge \omega_{B} \wedge J=\mathcal{K}_{A B C} v^{C}, \\
\mathcal{K}_{A} & =\int_{\mathrm{CY}_{3}} \omega_{A} \wedge J \wedge J=\mathcal{K}_{A B C} v^{B} v^{C}, \\
\mathcal{K} & =\int_{\mathrm{CY}_{3}} J \wedge J \wedge J=\mathcal{K}_{A B C} v^{A} v^{B} v^{C}=6 V_{\mathrm{CY}_{3}} .
\end{aligned}
$$

Using the following relation [66],

$$
* \omega_{A}=-J \wedge \omega_{A}+\frac{3}{2} \frac{\mathcal{K}_{A}}{\mathcal{K}} J \wedge J, \quad * J=\frac{1}{2} J \wedge J,
$$

we obtain

$$
\int_{\mathrm{CY}_{3}} \omega_{A} \wedge * \omega_{B}=-\mathcal{K}_{A B}+\frac{3}{2} \frac{\mathcal{K}_{A} \mathcal{K}_{B}}{\mathcal{K}} .
$$

Equation (D4) is related to gauge couplings of the vector multiplets in the low energy effective action of type IIA supergravity compactified on a Calabi-Yau threefold. If the NSNS 2-form $B_{2}$ vanishes, the matrix of the gauge couplings is given by [41-44]

$$
\begin{aligned}
\operatorname{Im} \mathcal{N}_{00} & =-\frac{\mathcal{K}}{6}=-V_{\mathrm{CY}_{3}}, \\
\operatorname{Im} \mathcal{N}_{A B} & =-\int_{\mathrm{CY}_{3}} \omega_{A} \wedge * \omega_{B} .
\end{aligned}
$$

Introducing $\mathcal{K}^{A B}$ by $\mathcal{K}^{A B} \mathcal{K}_{A C}=\delta^{A}{ }_{C}$, one derives the inverse matrix:

$$
\begin{aligned}
\left(\operatorname{Im} \mathcal{N}^{-1}\right)^{00} & =-\frac{6}{\mathcal{K}} \\
\left(\operatorname{Im} \mathcal{N}^{-1}\right)^{A B} & =-\int_{\mathrm{CY}_{3}} \tilde{\omega}^{A} \wedge * \tilde{\omega}^{B}
\end{aligned}
$$

where we defined the dual basis $\tilde{\omega}^{A} \in H^{2,2}\left(\mathrm{CY}_{3}\right)$ by

$$
\int_{\mathrm{CY}_{3}} \omega_{A} \wedge \tilde{\omega}^{B}=\delta_{A}^{B}
$$

and

$$
\begin{aligned}
\int_{\mathrm{CY}_{3}} \tilde{\omega}^{A} \wedge & * \tilde{\omega}^{B}=-\mathcal{K}^{A B}+\frac{3 v^{A} v^{B}}{\mathcal{K}} \\
* \tilde{\omega}^{A} & =\left(-\mathcal{K}^{A B}+\frac{3 v^{A} v^{B}}{\mathcal{K}}\right) \omega_{B}
\end{aligned}
$$

\section{APPENDIX E: HODGE DUAL OF $\left(\alpha_{\hat{K}}, \beta^{\hat{K}}\right)$}

$\left\{\alpha_{\hat{K}}, \beta^{\hat{K}}\right\}$ are both 3 -forms and those Hodge duals are also 3 -forms which can be expanded in terms of $\alpha_{\hat{K}}$ and $\beta^{\hat{K}}$ according to

$$
\begin{aligned}
& * \alpha_{\hat{K}}=A_{\hat{K}}^{\hat{L}} \alpha_{\hat{L}}+B_{\hat{K} \hat{L}} \beta^{\hat{L}}, \\
& * \beta^{\hat{K}}=C^{\hat{K} \hat{L}} \alpha_{\hat{L}}+D^{\hat{K}}{ }_{\hat{L}} \beta^{\hat{L}} .
\end{aligned}
$$

Using

$$
\int_{\mathrm{CY}_{3}} \alpha_{\hat{K}} \wedge \beta^{\hat{L}}=\delta_{\hat{K}}^{\hat{L}}
$$

one derives

$$
\begin{aligned}
B_{\hat{K} \hat{L}} & =\int_{\mathrm{CY}_{3}} \alpha_{\hat{K}} \wedge * \alpha_{\hat{L}}=\int_{\mathrm{CY}_{3}} \alpha_{\hat{L}} \wedge * \alpha_{\hat{K}}=B_{\hat{L} \hat{K}}, \\
-C^{\hat{K} \hat{L}} & =\int_{\mathrm{CY}_{3}} \beta^{\hat{K}} \wedge * \beta^{\hat{L}}=\int_{\mathrm{CY}_{3}} \beta^{\hat{L}} \wedge * \beta^{\hat{K}}=-C^{\hat{L} \hat{K}}, \\
-A_{\hat{K}}^{\hat{L}} & =\int_{\mathrm{CY}_{3}} \beta^{\hat{L}} \wedge * \alpha_{\hat{K}}=\int_{\mathrm{CY}_{3}} \alpha_{\hat{K}} \wedge * \beta^{\hat{L}}=D_{\hat{K}}^{\hat{L}} .
\end{aligned}
$$

The matrices $A, B, C, D$ are determined in terms of a matrix $\mathcal{M}[67,68]$

$$
\begin{aligned}
& A=(\operatorname{Re} \mathcal{M})(\operatorname{Im} \mathcal{M})^{-1}, \\
& B=-(\operatorname{Im} \mathcal{M})-(\operatorname{Re} \mathcal{M})(\operatorname{Im} \mathcal{M})^{-1}(\operatorname{Re} \mathcal{M}), \\
& C=(\operatorname{Im} \mathcal{M})^{-1},
\end{aligned}
$$

where

$$
\mathcal{M}_{\hat{K} \hat{L}}=\overline{\mathcal{F}}_{\hat{K} \hat{L}}+2 i \frac{(\operatorname{Im} \mathcal{F})_{\hat{K} \hat{M}} Z^{\hat{M}}(\operatorname{Im} \mathcal{F})_{\hat{L} \hat{N}} Z^{\hat{N}}}{Z^{\hat{M}}(\operatorname{Im} \mathcal{F})_{\hat{M} \hat{N}} Z^{\hat{N}}} .
$$

The matrix $\mathcal{M}$ satisfies

$$
\mathcal{F}_{\hat{K}}=\mathcal{M}_{\hat{K} \hat{L}} Z^{\hat{L}}
$$

where we have used the following relations on a holomorphic prepotential $\mathcal{F}$ with respect to $Z^{\hat{K}}$ :

$$
\mathcal{F}=\frac{1}{2} Z^{\hat{K}} \mathcal{F}_{\hat{K}}, \quad \mathcal{F}_{\hat{K}}=\frac{\partial \mathcal{F}}{\partial Z^{\hat{K}}}=Z^{\hat{K}} \mathcal{F}_{\hat{K} \hat{L}}, \quad \mathcal{F}_{\hat{K} \hat{L}}=\frac{\partial \mathcal{F}_{\hat{L}}}{\partial Z^{\hat{K}}} .
$$


[1] R. H. Brandenberger and C. Vafa, Nucl. Phys. B316, 391 (1989).

[2] T. Battefeld and S. Watson, Rev. Mod. Phys. 78, 435 (2006).

[3] R. H. Brandenberger, Mod. Phys. Lett. A 22, 1875 (2007).

[4] L. McAllister and E. Silverstein, Gen. Relativ. Gravit. 40, 565 (2008).

[5] R. H. Brandenberger, arXiv:0808.0746.

[6] D. Baumann and L. McAllister, arXiv:0901.0265.

[7] A. A. Tseytlin and C. Vafa, Nucl. Phys. B372, 443 (1992).

[8] S. Alexander, R. H. Brandenberger, and D. Easson, Phys. Rev. D 62, 103509 (2000).

[9] S. Watson and R. Brandenberger, J. Cosmol. Astropart. Phys. 11 (2003) 008.

[10] A. Kaya and T. Rador, Phys. Lett. B 565, 19 (2003).

[11] B. Bassett, M. Borunda, M. Serone, and S. Tsujikawa, Phys. Rev. D 67, 123506 (2003).

[12] T. Batterfeld and S. Watson, J. Cosmol. Astropart. Phys. 06 (2004) 001.

[13] S. Watson, Phys. Rev. D 70, 066005 (2004).

[14] S. P. Patil and R. Brandenberger, Phys. Rev. D 71, 103522 (2005).

[15] S. P. Patil and R. Brandenberger, J. Cosmol. Astropart. Phys. 01 (2006) 005.

[16] S. P. Patil, arXiv:hep-th/0504145.

[17] S. Kanno and J. Soda, Phys. Rev. D 72, 104023 (2005).

[18] R. Brandenberger, Y. K. Cheung, and S. Watson, J. High Energy Phys. 05 (2006) 025.

[19] A. Chatrabhuti, Int. J. Mod. Phys. A 22, 165 (2007).

[20] A. J. Berndsen and J. M. Cline, Int. J. Mod. Phys. A 19, 5311 (2004).

[21] A. Berndsen, T. Biswas, and J.M. Cline, J. Cosmol. Astropart. Phys. 08 (2005) 012.

[22] D. Easson and M. Trodden, Phys. Rev. D 72, 026002 (2005).

[23] S. Cremonini and S. Watson, Phys. Rev. D 73, 086007 (2006).

[24] R.J. Danos, A. R. Frey, and R.H. Brandenberger, Phys. Rev. D 77, 126009 (2008).

[25] M. Sano and H. Suzuki, Phys. Rev. D 78, 064045 (2008).

[26] G. Shiu and L-.T. Wang, Phys. Rev. D 69, 126007 (2004).

[27] S. S. Gubser and P. J. E. Peebles, Phys. Rev. D 70, 123510 (2004).

[28] S. S. Gubser and P. J. E. Peebles, Phys. Rev. D 70, 123511 (2004).

[29] A. Nusser, S. S. Gubser, and P. J. E. Peebles, Phys. Rev. D 71, 083505 (2005).

[30] T. Biswas, R. Brandenberger, A. Mazumdar, and T. Multamaki, Phys. Rev. D 74, 063501 (2006).

[31] J. Polchinski, String Theory (Cambridge University Press, Cambridge, England, 1998).

[32] E. Bergshoeff, B. Janssen, and T. Ortin, Phys. Lett. B 410, 131 (1997).
[33] E. Eyras, B. Janssen, and Y. Lozano, Nucl. Phys. B531, 275 (1998).

[34] E. Bergshoeff, Y. Lozano, and T. Ortin, Nucl. Phys. B518, 363 (1998).

[35] N. A. Obers and B. Pioline, Phys. Rep. 318, 113 (1999).

[36] K. Becker, M. Becker, and A. Strominger, Nucl. Phys. B456, 130 (1995).

[37] D. Joyce, arXiv:math/0111111.

[38] D. Joyce, Contemp. Math. 314, 125 (2002).

[39] A. Strominger, Nucl. Phys. B451, 96 (1995).

[40] B. de Wit, P. G. Lauwers, and A. Van Proeyen, Nucl. Phys. B255, 569 (1985).

[41] T. W. Grimm and J. Louis, Nucl. Phys. B718, 153 (2005).

[42] R. Boehm, H. Guenther, C. Herrmann, and Jan Louis, Nucl. Phys. B569, 229 (2000).

[43] J. Louis and A. Micu, Nucl. Phys. B635, 395 (2002).

[44] T. W. Grimm and J. Louis, Nucl. Phys. B699, 387 (2004).

[45] S. Watson and R. Brandenberger, J. High Energy Phys. 03 (2004) 045.

[46] S. Watson, Phys. Rev. D 70, 023516 (2004).

[47] S. B. Giddings, S. Kachru, and J. Polchinski, Phys. Rev. D 66, 106006 (2002).

[48] S. Kachru, R. Kallosh, A. Linde, and S. P. Trivedi, Phys. Rev. D 68, 046005 (2003).

[49] S. Kachru et al., J. Cosmol. Astropart. Phys. 10 (2003) 013.

[50] E. Silverstein, Phys. Rev. D 77, 106006 (2008).

[51] P. K. Townsend and M. N. R. Wohlfarth, Phys. Rev. Lett. 91, 061302 (2003).

[52] M. Sano and H. Suzuki, Phys. Rev. D 76, 064006 (2007).

[53] R. Brandenberger, D. A. Easson, and A. Mazumdar, Phys. Rev. D 69, 083502 (2004).

[54] T. Biswas, R. Brandenberger, D. A. Easson, and A. Mazumdar, Phys. Rev. D 71, 083514 (2005).

[55] N. Shuhmaher and R. Brandenberger, Phys. Rev. Lett. 96, 161301 (2006).

[56] T. Biswas and S. Alexander, arXiv:0812.3182.

[57] S. Bhowmick, S. Digal, and S. K. Rama, arXiv:0810.4049.

[58] F. Ferrer, T. Multamaki, and S. Rasanen, J. High Energy Phys. 04 (2009) 006.

[59] A. Arslanargin and A. Kaya, arXiv:0901.4608.

[60] T. Biswas and A. Mazumdar, arXiv:0901.4930.

[61] N. Ohta, Phys. Rev. Lett. 91, 061303 (2003).

[62] N. Ohta, Prog. Theor. Phys. 110, 269 (2003).

[63] N. Ohta, Int. J. Mod. Phys. A 20, 1 (2005).

[64] K. i. Maeda, N. Ohta, and K. Uzawa, J. High Energy Phys. 06 (2009) 051.

[65] C. M. Chen et al., J. High Energy Phys. 10 (2003) 058.

[66] A. Strominger, Phys. Rev. Lett. 55, 2547 (1985).

[67] H. Suzuki, Mod. Phys. Lett. A 11, 623 (1996).

[68] A. Ceresole, R. D’Auria, and S. Ferrara, Nucl. Phys. B, Proc. Suppl. 46, 67 (1996). 